\title{
THE COMPOSITION ALGEBRA OF A CYCLIC QUIVER
}

\author{
Towards an explicit description of the quantum group of type $\tilde{A}_{n}$
}

\section{CLAUS MICHAEL RINGEL}

[Received 26 April 1991]

\section{Introduction}

1.1. Let $k$ be a field. Let $\mathbb{T}=\mathbb{T}(n, k)$ be the category of locally nilpotent finite-dimensional representations over $k$ of the cyclic quiver $\Delta=\Delta(n)$ with $n \geqslant 2$ vertices:

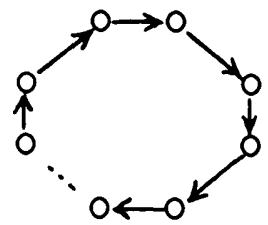

Note that $\Delta$ is also called the quiver of type $\tilde{A}_{n-1}$ with cyclic orientation. We denote by $\Delta_{0}$ the set of vertices of $\Delta$ (and often we will identify $\Delta_{0}$ with $\mathbb{Z} / n \mathbb{Z}$, or also with the set $\left\{x_{1}, x_{2}, \ldots, x_{n}\right\}$ or just with $\{1,2, \ldots, n\}$, with arrows $x_{i} \rightarrow x_{i+1}$ or $i \rightarrow i+1)$.

There are $n$ one-dimensional representations, corresponding to the vertices of $\Delta$; these are (up to isomorphism) all the simple objects of $T$. The simple representation corresponding to the vertex $a$ of $\Delta$ will be denoted by $S(a)$, and if $S^{\prime}$ is isomorphic to $S(a)$, we will write $\left[S^{\prime}\right]=a$. Given a simple representation $S$, and $l \in \mathbb{N}_{1}$, there is (up to isomorphism) a unique indecomposable representation $S[l]$ of length $l$ with top $S$, and we obtain in this way all indecomposable representations (again up to isomorphism). It follows that we can index the isomorphism classes in $\mathbb{T}$ by the set $\Pi$ of $n$-tuples of partitions; the representation of $\Delta$ corresponding to $\pi \in \Pi$ will be denoted by $M(\pi)$; see $\S 3.3$.

1.2. The elements of $\Delta_{0}$ may be considered as letters in order to form words, and we denote by $\Omega=\Omega\left(\Delta_{0}\right)$ the set of words in these letters. Given a composition series

$$
M=F_{0} \supset F_{1} \supset \ldots \supset F_{m}=0
$$

of some representation $M$ of $\Delta$, we may form the word

$$
\omega(F)=\left[F_{0} / F_{1}\right]\left[F_{1} / F_{2}\right] \ldots\left[F_{m-1} / F_{m}\right]
$$

in $\Omega$, and call it the type of the composition series $F$. Given $w \in \Omega$ and $\pi \in \Pi$, there is a polynomial $\langle w, \pi\rangle \in \mathbb{Z}[q]$ (the polynomial ring with integer coefficients in the variable $q$ ) such that for finite fields $k$, the evaluation of $\langle w, \pi\rangle$ at the cardinality $|k|$ of $k$ yields the number of composition series of $M(\pi)$ of type $w$; see $\S 8.1$.

1991 Mathematics Subject Classification: primary 16G20, 05A10, 17B67, 17B37; secondary 16P10, 05A30, 68R15, 81R50.

Proc. London Math. Soc. (3) 66 (1993) 507-537. 
Let $\mathscr{A}$ be the localization of the polynomial ring $\mathbb{Q}[q]$ at the maximal ideal generated by $q-1$, and consider the free $\mathscr{A}$-algebra generated by $\Delta_{0}$; it is just the semigroup algebra $\mathscr{A} \Omega$ of $\Omega$ over $\mathscr{A}$. Similarly, we may consider the free $\mathscr{A}$-module with basis $\Pi$, and we may extend $\langle-,-\rangle$ to an $\mathscr{A}$-bilinear map

$$
\langle-,-\rangle: \mathscr{A} \Omega \times \mathscr{A} \Pi \rightarrow \mathscr{A} \text {. }
$$

Let $\mathscr{R}$ be the set of elements $x \in \mathscr{A} \Omega$ such that $\langle x,-\rangle=0$; similarly, let $\mathscr{S}$ be the set of all elements $y \in \mathscr{A} \Pi$ such that $\langle-, y\rangle=0$.

1.3. As in [7], we consider the polynomials

$$
\begin{aligned}
& \rho_{1}(X, Y)=Y X-X Y, \\
& \rho_{2}^{+}(X, Y)=Y X^{2}-(q+1) X Y X+q X^{2} Y, \\
& { }^{+} \rho_{2}(X, Y)=X^{2} Y-(q+1) X Y X+q Y X^{2}
\end{aligned}
$$

in two non-commuting variables $X, Y$ over $\mathbb{Z}[q]$, and also

$$
\rho_{3}(X, Y)=Y X^{3}-\left(q+1+q^{-1}\right) X Y X^{2}+\left(q+1+q^{-1}\right) X^{2} Y X-X^{3},
$$

over $\mathbb{Z}\left[q, q^{-1}\right]$.

In case $n=2$, let $\mathscr{R}^{\prime}$ be the ideal of $\mathscr{A} \Omega$ generated by

$$
\rho_{3}\left(x_{1}, x_{2}\right), \rho_{3}\left(x_{2}, x_{1}\right),
$$

whereas for $n \geqslant 3$, let $\mathscr{R}^{\prime}$ be the ideal of $\mathscr{A} \Omega$ generated by

$$
{ }^{+} \rho_{2}\left(x_{i}, x_{i+1}\right)=0, \quad \rho_{2}^{+}\left(x_{i+1}, x_{i}\right)=0,
$$

for all $1 \leqslant i \leqslant n$, and by $\rho_{1}\left(x_{i}, x_{j}\right)=0$ for all $j \not \equiv i \pm 1(\bmod n)$.

THEOREM A. $\mathscr{R}=\mathscr{R}^{\prime}$.

We will call $\mathscr{C}=\mathscr{C}(n)=\mathscr{A} \Omega / \mathscr{R}$ the composition algebra of $\Delta$, since $\mathscr{R}$ just exhibits all the relations which are universally valid for composition series of representations of $\Delta$.

Of course, the polynomials defining $\mathscr{R}^{\prime}$ are strongly related to (and for $n=2$ identical with) the Drinfeld-Jimbo relations for the quantization $U\left(\mathfrak{n}_{+}\right)$of the universal enveloping algebra of $\mathfrak{n}_{+}$, where $\mathfrak{g}=\mathfrak{n}_{-} \oplus \mathfrak{h} \oplus \mathfrak{n}_{+}$is a triangular decomposition of the Kac-Moody algebra of type $\tilde{A}_{n-1}$.

The corresponding result for $\mathfrak{g}$ a finite-dimensional semisimple complex Lie algebra presented in [7] gave immediately a convenient description of $U\left(\mathfrak{h} \oplus \mathfrak{n}_{+}\right)$, since in this case $\mathscr{C}$ is just the generic Hall algebra with coefficients in $\mathscr{A}$, and therefore the free $\mathscr{A}$-module on the set of isomorphism classes of representations of a corresponding quiver or species. Also in the case studied here, we will see that $\mathscr{C}$ is a free $\mathscr{A}$-module, and we are going to exhibit explicitly a basis of $\mathscr{C}$.

1.4. An $n$-tuple $\pi=\left(\pi^{(1)}, \ldots, \pi^{(n)}\right)$ of partitions will be called separated, provided for every integer $h \geqslant 1$, the dual of at least one of the partitions $\pi^{(1)}, \ldots, \pi^{(n)}$ has no part of length $h$. Let $\Pi^{s}$ be the set of separated $n$-tuples of partitions; this will be our basic index set. For $\pi \in \Pi^{s}$, we will define a word $\omega_{\pi} \in \Omega$; see $\S 4.4$. 
THEOREM B. The set of elements $\omega_{\pi}$, with $\pi \in \Pi^{s}$, is the basis of a free $\mathscr{A}$-submodule of $\mathscr{A} \Omega$, and this submodule is a direct complement for $\mathscr{R}$.

Of course, this means that the residue classes modulo $\mathscr{R}$ of the elements $\omega_{\pi}$, with $\pi \in \Pi^{s}$, form a free $\mathscr{A}$-basis for $\mathscr{C}$. Also, we should remark that the set $\Pi^{s}$ itself is the basis of a free $\mathscr{A}$-submodule of $\mathscr{A} \Pi$ which is a direct complement of $\mathscr{S}$.

1.5. The main device in our consideration is a partial ordering $\leqslant$ on $\Pi$, which corresponds to elementary degenerations in $\pi$.

THEOREM C. Let $\pi \in \Pi^{s}$. A representation $M$ of $\Delta$ has a composition series of type $\omega_{\pi}$ if and only if $M$ is isomorphic to some $M(\lambda)$ with $\lambda \leqslant \pi$.

The number of composition series of $M(\pi)$ of type $\omega_{\pi}$ can easily be exhibited. Given a word $w=s_{1}^{e_{1}} \ldots s_{m}^{e_{m}}$ with letters $s_{i} \in \Delta_{0}, s_{i} \neq s_{i+1}$, and $e_{i} \geqslant 1$ for all $i$, consider the polynomial

$$
r(w)=\prod_{i=1}^{m} \psi_{e_{i}}(q), \quad \text { where } \psi_{e}(q)=\frac{(1-q)\left(1-q^{2}\right) \ldots\left(1-q^{e}\right)}{(1-q)^{e}}
$$

in $\mathbb{Z}[q]$.

Theorem D. Let $\pi \in \Pi^{s}$. Then $\left\langle\omega_{\pi}, \pi\right\rangle=r\left(\omega_{\pi}\right)$.

1.6. The proof of these results will rely on combinatorial considerations dealing with words and with $n$-tuples of partitions. Given an $n$-tuple of partitions, we will have to use $\pi$-tableaux: as usual, we visualize partitions by diagrams made up from squares, and now we label these squares by consecutive integers. Of particular interest will be the so-called column-increasing $\pi$-tableaux, since they correspond to composition series of $M(\pi)$. The reduction from representations and composition series to tableaux will be given in $\S 3$.

The results presented here have been reported at the Bad Honnef workshop, January 1990, the Tsukuba conference on Representation Theory of Algebras, August 1990, and at the Scuola Normale Superiore in Pisa, March 1991. The author is indebted to these institutions for their kind hospitality.

\section{Composition series}

In dealing with an object $M$ in a length category, we may consider the various composition series of $M$. As in the case of the category of representations of the cyclic quiver, we may define the type of any composition series of $M$ to indicate the consecutive composition factors.

2.1. Words. Let $\Delta_{0}$ be any set. We denote by $\Omega=\Omega\left(\Delta_{0}\right)$ the free semigroup with unit element, generated by $\Delta_{0}$. The elements of $\Omega$ will be called words; there is the empty word 1 , and the other words are of the form $w=s_{1} s_{2} \ldots s_{m}$ with $s_{i} \in \Delta_{0}$ for $1 \leqslant i \leqslant m$, and $m \geqslant 1$. Any word has a length, the length of 1 being 0 , whereas the length of $w=s_{1} s_{2} \ldots s_{m}$ with $s_{i} \in \Delta_{0}$ is $m$. If $w=s_{1} s_{2} \ldots s_{m}$, 
with $s_{i} \in \Delta_{0}$, then $\{1,2, \ldots, m\}$ will be called the support supp $w$ of $w$, and for $I \subseteq \operatorname{supp} w$, say $I=\left\{i_{1}<i_{2}<\ldots<i_{t}\right\}$, we define $w \mid I=s_{i_{1}} s_{i_{2}} \ldots s_{i_{i}}$, and call $w \mid I$ a subword of $w$, and $w \mid C I$ a complementary subword for $w \mid I$ in $w$, where $\mathbf{C} I=\operatorname{supp} w \backslash I$. (Note that a subword of $w$ may have several complementary subwords, depending on its embedding: for example, let $\Delta_{0}=\{1,2,3\}$ and $w=1232$. Then $w^{\prime}=12$ can be realized as $w \mid\{1,2\}$ with complementary subword 32 , and as $w \mid\{1,4\}$, with complementary subword 23 .)

Let $\Omega^{\prime}, \Omega^{\prime \prime}$ be two subsets of $\Omega$. The merging $\Omega^{\prime} \times \Omega^{\prime \prime}$ of $\Omega^{\prime}$ and $\Omega^{\prime \prime}$ is the subset

$$
\Omega^{\prime} \times \Omega^{\prime \prime}=\left\{w \in \Omega \mid \text { there is } I \subseteq \operatorname{supp} w \text { with } w\left|I \in \Omega^{\prime}, w\right| \mathbf{C} I \in \Omega^{\prime \prime}\right\} .
$$

Clearly, this is a commutative and associative operation on the set of subsets of $\Omega$.

2.2. The types of composition series. Let $\mathbb{L}$ be an abelian category. The isomorphism class of an object $M$ will be denoted by [M]. The direct sum of $m$ copies of $M$ will be denoted by $m M$. Recall that $\mathbb{L}$ is called a length category provided every object in $\mathbb{Q}$ has a (finite) composition series. Now, assume that $\mathbb{L}$ is a length category, and let $\Delta_{0}=\Delta_{0}(\mathbb{L})$ be the set of isomorphism classes of simple objects in $\mathbb{L}$. (In case $\mathbb{L}=\mathbb{T}(n, k)$, the notation is the same as in the Introduction). Given an object $M$ in $\mathbb{Q}$ with a composition series $M=F_{0} \supset F_{1} \supset \ldots \supset F_{m}=0$, then

$$
\omega(F)=\left[F_{0} / F_{1}\right]\left[F_{1} / F_{2}\right] \ldots\left[F_{m-1} / F_{m}\right]
$$

is called the type of $F$; it is an element of $\Omega=\Omega\left(\Delta_{0}\right)$. We denote by $\Omega_{M}$ the set of types of composition series of $M$. Of course, the zero module $M=0$ has precisely one composition series, and its type is the empty word $1 \in \Omega\left(\Delta_{0}\right)$.

Lemma 1. Let $U$ be a subobject of $M$. Then

$$
\Omega_{M} \subseteq \Omega_{U} \times \Omega_{M / U} .
$$

Lemma 2. Let $M, M^{\prime}$ be objects in $\mathbb{L}$. Then

$$
\Omega_{M \oplus M^{\prime}}=\Omega_{M} \times \Omega_{M^{\prime}} .
$$

Proof. Let $F$ be a composition series of $M$ of type $w=s_{1} s_{2} \ldots s_{m}$, say

$$
M=F_{0} \supset F_{1} \supset \ldots \supset F_{m}=0,
$$

where $s_{i}=\left[F_{i-1} / F_{i}\right]$. Let $U$ be a subobject of $M$, and consider the filtration

$$
U=F_{0} \cap U \supseteq F_{1} \cap U \supseteq \ldots \supseteq F_{m} \cap U=0 .
$$

Let $I=\left\{i \mid 1 \leqslant i \leqslant m, F_{i-1} \cap U \neq F_{i} \cap U\right\}$. Then $w \mid I$ is the type of the composition series of $U$ induced by $F$, and $w \mid C I$ is the type of the composition series of $M / U$ induced by $F$. This yields Lemma 1 , and thus also the inclusion

$$
\Omega_{M \oplus M^{\prime}} \subseteq \Omega_{M} \times \Omega_{M^{\prime}}
$$

in Lemma 2. For the other inclusion, let $w \in \Omega$, and let $I \subseteq \operatorname{supp} w=$ $\{1,2, \ldots, m\}$, such that there is a filtration $F$ of $M$ of type $w \mid I$, and a filtration $F^{\prime}$ of $M^{\prime}$ of type $w \mid \mathbf{C l}$. We define a filtration $F^{\prime \prime}$ of $M \oplus M^{\prime}$ inductively as follows. Let $F_{0}^{\prime \prime}=F_{0} \oplus F_{0}^{\prime}=M \oplus M^{\prime}$. If $F_{t}^{\prime \prime}=F_{i} \oplus F_{t-i}^{\prime}$ is already defined for some 
$0 \leqslant t<m$, and $t+1 \in I$, let $F_{t+1}^{\prime \prime}=F_{i+1} \oplus F_{t-i}^{\prime}$, whereas for $t+1 \notin I$, let $F_{t+1}^{\prime \prime}=$ $F_{i} \oplus F_{t-i+1}^{\prime}$. Clearly, $F^{\prime \prime}$ is a composition series of $M \oplus M^{\prime}$ of type $w$.

2.3. Reduced filtrations. Let us assume that for any simple object $S$ in $\mathbb{L}$ we have $\operatorname{Ext}^{1}(S, S)=0$. In this case, it seems to be convenient to consider besides composition series also certain filtrations with homogeneous semisimple factors. Let $w=s_{1}^{e_{1}} s_{2}^{e_{2}} \ldots s_{m}^{e_{m}}$ with $s_{i} \in \Delta_{0}, e_{i} \in \mathbb{N}_{1}$, and $s_{i-1} \neq s_{i}$ for all $1<i \leqslant m$. A reduced filtration $G$ of an object $M$ of $\mathbb{L}$ of type $w$ is a filtration

$$
M=G_{0} \supset G_{1} \supset \ldots \supset G_{m}=0
$$

such that $G_{i-1} / G_{i} \cong e_{i} S_{i}$, where $\left[S_{i}\right]=s_{i}$. Note that any reduced filtration of type $w$ can be refined to a composition series of type $w$, and conversely, given a composition series $F$ of type $w$, then there is a unique reduced filtration $G$ of type $w$ such that $F$ is a refinement of $G$ : let $u_{t}=\sum_{i=1}^{t} e_{i}$, and $G_{t}=F_{u_{t}}$, for $0 \leqslant t \leqslant m$; this is a reduced filtration of type $w$, since we assume that the simple objects have no self-extensions.

\section{Reduction}

The problem we are interested in is to describe the possible types of composition series of modules in $\mathbb{T}(n, k)$. Here, we will reduce this problem to a purely combinatorial one.

3.1. The diagram of a partition. We recall some well-known definitions. A partition is a decreasing sequence $p=\left(p_{1}, p_{2}, p_{3}, \ldots\right)$ of non-negative integers with finite support; thus $p_{1} \geqslant p_{2} \geqslant \ldots \geqslant p_{m} \geqslant p_{m+1} \geqslant \ldots$, and $p_{m}=0$ for $m \gg 0$. The numbers $p_{i}$ are the parts of $p$; we say that the ith part is of length $p_{i}$. If $p_{m+1}=0$, we will usually write just $p=\left(p_{1}, p_{2}, \ldots, p_{m}\right)$; thus we identify finite sequences which only differ by adding some zeros at the end. The zero partition $(0,0, \ldots)$ will be denoted by $(0)$. The number of non-zero parts of $p$ will be called the height of $p$. Given a partition $p$, we denote by $\tilde{p}$ the dual partition (thus, $\bar{p}_{i}$ is the number of parts of $p$ whose length is at least $i$ ); in particular, $\tilde{p}_{1}$ is the height of $p$.

Recall that partitions are usually visualized by drawing corresponding (Ferrers or Young) diagrams: if $p=\left(p_{1}, p_{2}, \ldots\right)$ is a partition, let

$$
\|p\|=\left\{(i, j) \mid 1 \leqslant j \leqslant p_{i}, 1 \leqslant i\right\} ;
$$

in drawing such diagrams, one usually adopts the convention that the first coordinate $i$ increases as one goes downwards, and the second coordinate increases as one goes from left to right, and one replaces the nodes by squares; thus, for example, the partition $(5,4,4,1)$ is depicted as

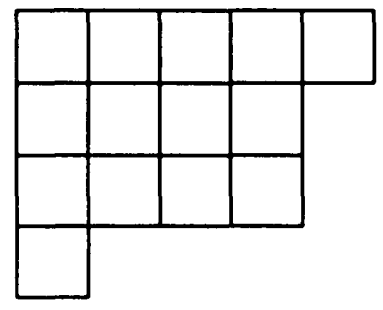


The reason for using squares is the following: often it will be necessary to label the nodes by letters or integers and it is convenient to write the labels into the squares.

Let us consider now $n$-tuples of partitions. For $\pi \in \Pi$, its diagram is given by

$$
\|\pi\|=\left\{(a, i, j) \mid 1 \leqslant j \leqslant \pi_{i}^{(a)}, 1 \leqslant i, 1 \leqslant a \leqslant n\right\},
$$

and we call the elements $(a, i, j)$ the squares of $\|\pi\|$. The numbers $a, i, j$ of $(a, i, j)$ will be referred to as the partition index, the row index, and the column index, respectively. For any square $(a, i, j)$ of $\|\pi\|$, we introduce its type $s(a, i, j)=\overline{a+i-1}$, here $\bar{a}$ denotes the residue class of $a \in \mathbb{Z}$ modulo $n$. In the partition $\pi^{(a)}$, the squares in the first row have type $a$, those in the second have type $\overline{a+1}$, and so on; in general, the squares in the $i$ th row are of type $\overline{a+i-1}$.

3.2. Tableaux. Let $\pi$ be an $n$-tuple of partitions and let $|\pi|=\sum_{a, i} \pi_{i}^{(a)}$; this is called the length of $\pi$. A bijective map $T:\|\pi\| \rightarrow\{1,2, \ldots,|\pi|\}$ is called a $\pi$-tableau. (Sometimes, it will be convenient to allow as the value set an arbitrary totally ordered set consisting of $|\pi|$ elements, instead of the canonical one $\{1,2, \ldots,|\pi|\}$.) A $\pi$-tableau yields a total ordering of the squares of the diagram $\|\pi\|$ of $\pi$. Given a $\pi$-tableau $T$, we define a word $\omega(T) \in \Omega(\mathbb{Z} / n \mathbb{Z})$ by

$$
\omega(T)=s\left(T^{-1}(1)\right) s\left(T^{-1}(2)\right) \ldots s\left(T^{-1}(|\pi|)\right),
$$

where $s:\|\pi\| \rightarrow \mathbb{Z} / n \mathbb{Z}$ is the type function. A $\pi$-tableau $T$ is said to be column-increasing in the case where $T(a, i, j)<T(a, i+1, j)$ for all $(a, i, j)$, $(a, i+1, j) \in\|\pi\|$. Similarly, $T$ is said to be row-increasing in the case where $T(a, i, j)<T(a, i, j+1)$ for all $(a, i, j),(a, i+1, j) \in\|\pi\|$. Finally, $T$ is said to be standard provided it is both column-increasing and row-increasing.

3.3. Tubes. A length category $T$ will be called a tube of rank $n$ provided gl. $\operatorname{dim} \mathbb{T}=1$, and there are precisely $n$ (isomorphism classes of) simple objects $S(1), \ldots, S(n)$ such that

$$
\operatorname{Ext}^{1}(S(i), S(j))=0 \quad \text { for } j \not \equiv i+1(\bmod n),
$$

whereas $\operatorname{Ext}^{1}(S(i), S(i+1))$ is one-dimensional both as a left End $S(i)$-space as well as a right End $S(i+1)$-space, for $1 \leqslant i \leqslant n$, and $S(n+1)=S(1)$. In particular, $\Delta(\mathbb{T})$ can be identified with $\mathbb{Z} / n \mathbb{Z}$, or with $\{1,2, \ldots, n\}$.

Note that the category $\mathbb{T}(n, k)$ of locally nilpotent representations over $k$ of the cyclic quiver $\Delta(n)$ is a tube of rank $n$. It is well-known that any tube may be considered as the category of finite length modules over some ring; thus we will not mind calling the objects in $\mathbb{T}$ modules. In particular, the category $\mathbb{T}(n, k)$ is equivalent to the category of finite length modules over the $n \times n$-matrix ring

$$
\left.\left[\begin{array}{cccc}
\mathcal{O} & \mathcal{O} & \cdots & \mathcal{O} \\
\langle X\rangle & \mathcal{O} & \cdots & \mathcal{O} \\
\vdots & \ddots & \ddots & \vdots \\
\langle X\rangle & \cdots & \langle X\rangle & \mathcal{O}
\end{array}\right]=\left\{\left(a_{i j}\right)_{i j} \in M_{n}(\mathcal{O}) \mid a_{i j} \in\langle X\rangle \text { for } i\right\rangle j\right\}
$$

where $\mathcal{O}=k[[X]]$ is the power-series ring over $k$ in one variable $X$, and $\langle X\rangle$ is the ideal generated by $X$; thus $\langle X\rangle$ is the maximal ideal of $\mathcal{O}$. 
Let $\mathbb{T}$ be a tube of rank $n$. Any indecomposable module in $\mathbb{T}$ is serial: it has a unique composition series. Given a simple module $S$ and a natural number $l \in \mathbb{N}_{1}$, there is (up to isomorphism) a unique indecomposable module $S[l]$ of length $l$ with top $S$. Thus we can index the isomorphism classes of modules in $T$ by the set $\Pi$ of $n$-tuples of partitions, as follows: given partitions $\pi^{(1)}, \ldots, \pi^{(n)}$, let

$$
M(\pi)=\bigoplus_{a=1}^{n} \bigoplus_{j} S(a)\left[\tilde{\pi}_{j}^{(a)}\right],
$$

where $\tilde{\pi}^{(a)}=\left(\tilde{\pi}_{1}^{(a)}, \tilde{\pi}_{2}^{(a)}, \ldots\right)$ denotes the partition dual to $\pi^{(a)}$.

(The reason for invoking dual partitions is the following: the usual visualization of a partition $\pi^{(a)}$ depicts the parts of $\pi^{(a)}$ as rows of $\left\|\pi^{(a)}\right\|$, and the parts of $\tilde{\pi}^{(a)}$ as columns of $\left\|\pi^{(a)}\right\|$. If we want to interpret a partition as a direct sum of serial modules, it seems appropriate to consider the various columns as serial modules. More precisely, we consider the squares of any column as consecutive composition factors: the upper square of a column will correspond to the top composition factor, and so on, the lowest square of a column will correspond to the socle of the module. In this way, the vertical structure of a column agrees with the usual convention of drawing modules. Note however that Macdonald [5] uses the opposite rule: for him, the indecomposable modules correspond to the parts of a partition, they rest, apparently, with the top to the right and the socle to the left.)

3.4. Proposition 1. Let $\pi \in \Pi, w \in \Omega$. There exists a composition series of $M(\pi)$ of type $w$ if and only if there exists a column-increasing $\pi$-tableau $T$ with $\omega(T)=w$.

Before we start with the proof, let us consider the problem of equivalence of $\pi$-tableaux.

3.5. Equivalence of $\pi$-tableaux. Let $\pi$ be an $n$-tuple of partitions. Two $\pi$-tableaux $T_{1}, T_{2}$ are said to be equivalent provided the following conditions are satisfied: first, $\omega\left(T_{1}\right)=\omega\left(T_{2}\right)$, and, second, if we write $\omega\left(T_{1}\right)=s_{1}^{e_{1}} s_{2}^{e_{2}} \ldots s_{m}^{e_{m}}$ with $s_{i} \in \mathbb{Z} / n \mathbb{Z}, e_{i} \in \mathbb{N}_{1}$, and $s_{i} \neq s_{i+1}$ for all $i$, then there is a permutation $\sigma \in$ $\Sigma_{e_{1}} \times \Sigma_{e_{2}} \times \ldots \times \Sigma_{e_{m}}$ such that $T_{2}=\sigma \circ T_{1}$.

3.6. Proposition 2. Let $\pi \in \Pi, w \in \Omega$. Then all column-increasing $\pi$-tableaux $T$ with $\omega(T)=w$ are equivalent if and only if there exists at most one reduced filtration of $M(\pi)$ of type $w$.

We are going to present the proof of both propositions in this section.

3.7. Let $\pi \in \Pi$. Given a column-increasing $\pi$-tableau $T$, we define a composition series $F(T)$ of $M(\pi)$ with type $\omega(T)$.

As usual, we denote the radical of a module $M$ by $\operatorname{rad} M$; it is the intersection of the maximal submodules. The modules $S(a)[l]$, where $1 \leqslant a \leqslant n, l \geqslant 1$, are serial; in particular, $\operatorname{rad} S(a)[l]$ is the unique maximal submodule; note that $\operatorname{rad} S(a)[l]=S(\overline{a+1})[l-1]$, for $l \geqslant 2$, and $\operatorname{rad} S(a)[1]=0$. The submodules of $S(a)[l]$ are $\operatorname{rad}^{t} S(a)[l]$, with $0 \leqslant t \leqslant l$.

Let $\pi \in \Pi$. A subset $V \subseteq\|\pi\|$ will be called closed provided it satisfies the following property: if $(a, i, j) \in V,(a, i+1, j) \in\|\pi\|$, then $(a, i+1, j) \in V$. Let $V$ 
be a closed subset of $\|\pi\|$. For $1 \leqslant a \leqslant n$, and $1 \leqslant j \leqslant \tilde{\pi}_{1}^{(a)}$, let $h_{V}(a, j)=h(a, j)$ be the maximal number $i$ such that $(a, i, j) \notin V$ (in the case where $(a, 1, j) \in V$, we set $h(a, j)=0)$. We define

$$
U(V)=\bigoplus_{a=1}^{n} \bigoplus_{j} \operatorname{rad}^{h(a, j)} S(a)\left[\tilde{\pi}_{j}^{(a)}\right] \subseteq M(\pi) ;
$$

this is a submodule of $M(\pi)$ of length $|V|$.

Let $T$ be a $\pi$-tableau. Clearly, $T$ is column-increasing if and only if all the sets $V(T, u)=T^{-1}\{v|u<v \leqslant| \pi \mid\}$ with $0 \leqslant u \leqslant|\pi|$, are closed subsets of $\|\pi\|$. Consider now the case of $T$ being column-increasing. Let

$$
F(T)_{u}=U(V(T, u)) .
$$

Then $F(T)$ is a composition series of $M(\pi)$, and we claim that its type is $\omega(T)$. For, let $1 \leqslant u \leqslant|\pi|$, and $T(a, i, j)=u$. Then $h_{V(T, u-1)}(a, j)=i-1, h_{V(T, u)}(a, j)=$ $i$, and therefore

$$
\begin{aligned}
F(T)_{u-1} / F(T)_{u} & =U(V(T, u-1)) / U(V(T, u)) \\
& \cong \operatorname{rad}^{i-1} S(a)\left[\tilde{\pi}_{j}^{(a)}\right] / \operatorname{rad}^{i} S(a)\left[\tilde{\pi}_{j}^{(a)}\right] \\
& \cong S(a+i-1) .
\end{aligned}
$$

On the other hand, we also have

$$
s\left(T^{-1}(u)\right)=s(a, i, j)=\overline{a+i-1} .
$$

3.8. For $\pi \in \Pi$, there are always column-increasing $\pi$-tableaux. One particular example, $T_{\pi}$, is defined as follows: let

$$
T_{\pi}(a, i, j)=\sum_{a^{\prime}<a}\left|\pi^{\left(a^{\prime}\right)}\right|+\sum_{j^{\prime}<j} \tilde{\pi}_{j^{\prime}}^{(a)}+i
$$

(we arrange the columns by ordering the pairs $(a, j)$ lexicographically, and we number the columns one after the other, from top to socle; thus we deal with the lexicographical ordering with respect to considering first the partition-index, then the column-index, and finally the row-index).

3.9. Let $\pi \in \Pi$. Given a column-increasing $\pi$-tableau $T$, and a composition series $F$ of $M(\pi)$, we define a column-increasing $\pi$-tableau $\tau(T, F)$, such that $\omega(\tau(T, F))=\omega(F)$.

According to $\S 3.7$, there is defined a composition series $F(T)$. The comparison of the two composition series $F(T)$ and $F$ yields a permutation $\sigma$ of $\{1,2, \ldots,|\pi|\}$ such that

$$
F(T)_{u-1} / F(T)_{u} \cong F_{\sigma(u)-1} / F_{\sigma(u)},
$$

and we define $\tau(T, F)=\sigma \circ T$. More precisely, let $\sigma(u)$ be minimal with

$$
F_{\sigma(u)} \cap F(T)_{u-1} \subseteq F(T)_{u},
$$

or, equivalently, let $\sigma(u)$ be maximal with

$$
F(T)_{u}+F_{\sigma(u)-1} \supseteq F(T)_{u-1} .
$$

Then $\sigma$ is a permutation, and (*) is satisfied, according to the Jordan-Hölder theorem. It follows that the $\sigma(u)$ th letter in the type $\omega(F)$ of $F$ is 
$\left[F_{\sigma(u)-1} / F_{\sigma(u)}\right]=\left[F(T)_{u-1} / F(T)_{u}\right]$, but $\omega(F(T))=\omega(T)$, and thus this letter is $s\left(T^{-1}(u)\right)$. On the other hand, the $\sigma(u)$ th letter of $\omega(\tau(T, F))$ is

$$
s\left((\sigma \circ T)^{-1}(\sigma(u))\right)=s\left(T^{-1}(u)\right) .
$$

This shows that $\omega(\tau(T, F))=\omega(F)$.

3.10. Proof of Proposition 1. Let $\pi \in \Pi, w \in \Omega$. If $T$ is a column-increasing $\pi$-tableau with $\omega(T)=w$, then $\S 3.7$ shows that $F(T)$ is a composition series of $M(\pi)$ of type $w$. Conversely, if $F$ is a composition series of $M(\pi)$ of type $w$, then $\S 3.9$ shows that $\tau\left(T_{\pi}, F\right)$ is a column-increasing $\pi$-tableau with $\omega\left(\tau\left(T_{\pi}, F\right)\right)=w$.

3.11. Proof of Proposition 2. Let $\pi \in \Pi$ and $w \in \Omega$, say $w=s_{1}^{e_{1}} s_{2}^{e_{2}} \ldots s_{m}^{e_{m}}$ where $s_{i} \in \Delta_{0}, e_{i} \in \mathbb{N}_{1}$ and $s_{i-1} \neq s_{i}$ for all $1<i \leqslant m$. Let $u_{t}=\sum_{i=1}^{t} e_{i}$, for $0 \leqslant t \leqslant m$. First, assume that there exists only one reduced filtration $G$ of $M(\pi)$ of type $w$, and let $T, T^{\prime}$ be $\pi$-tableaux with $\omega(T)=\omega\left(T^{\prime}\right)=w$. The composition series $F(T)$ and $F\left(T^{\prime}\right)$ are both refinements of $G$; thus we have

$$
F(T)_{u_{t}}=G_{t}=F\left(T^{\prime}\right)_{u_{i}}
$$

for $0 \leqslant t \leqslant m$. But this means that $V\left(T, u_{t}\right)=V\left(T^{\prime}, u_{t}\right)$ for all $t$, and therefore $T$ and $T^{\prime}$ are equivalent.

Conversely, assume that all column-increasing $\pi$-tableaux $T$ with $\omega(T)=w$ are equivalent. Let $G, G^{\prime}$ be reduced filtrations of $M(\pi)$ of type $w$. Let $F$ be a composition series which refines $G$, and $T=\tau\left(T_{\pi}, F\right)$. Then $T$ is a columnincreasing $\pi$-tableau and $\omega(T)=w$. By reversed induction, we show that $F(T)_{u_{t}}=G_{t}^{\prime}$, for $t=m, m-1, \ldots, 0$. Nothing has to be shown for $t=m$. Assume we know that $F(T)_{u_{t}}=G_{t}^{\prime}$ for some $t$, and suppose $F(T)_{u_{t-1}} \neq G_{t-1}^{\prime}$. We refine $G^{\prime}$ to a composition series $F^{\prime}$ of $M(\pi)$ such that $F(T)_{u_{t-1}} \cap F_{u_{t}-1}^{\prime}=F(T)_{u_{i}}$. Consider now the $\pi$-tableau $\tau\left(T, F^{\prime}\right)$. By definition, $\tau\left(T, F^{\prime}\right)=\sigma \circ T$ for some permutation $\sigma$, and since both $T$ and $\tau\left(T, F^{\prime}\right)$ are column-increasing $\pi$-tableaux with $\omega(T)=w=\omega\left(\tau\left(T, F^{\prime}\right)\right)$, the permutation $\sigma$ belongs to $S_{e_{1}} \times S_{e_{2}} \times \ldots \times S_{e_{m}}$. Let $u=\sigma^{-1}\left(u_{t}\right)$. Then $u_{t-1}<u \leqslant u_{t}$. By the definition of $\sigma$, we see that $u_{t}(=\sigma(u))$ is minimal with

$$
F_{u_{t}}^{\prime} \cap F(T)_{u-1} \subseteq F(T)_{u}
$$

However, we know that

$$
F_{u_{t}-1}^{\prime} \cap F(T)_{u-1} \subseteq F_{u_{t}-1}^{\prime} \cap F(T)_{u_{t-1}}=F(T)_{u_{t}} \subseteq F(T)_{u},
$$

and this contradicts the minimality of $u_{t}$. This contradiction shows that $F(T)_{u_{t-1}}=$ $G_{t-1}^{\prime}$ and finishes the induction proof. In particular, for $G=G^{\prime}$, we see that also $F(T)_{u_{t}}=G_{t}$, for all $t$; thus there is just one reduced filtration of $M(\pi)$ of type $w$.

\section{Index sets}

We are going to exhibit some sets which will be needed as index sets in our investigation. We use two different central concepts, namely words and tuples of partitions, since our aim is to study composition series of serial modules: whereas the type of a composition series is a word (in $n$ letters, where $n$ is the number of isomorphism classes of simple modules), the isomorphism classes of serial modules are indexed by $n$-tuples of partitions. Here, we will formulate the basic definitions both for words and for tuples of partitions, and relate these concepts. 
We fix some natural number $n \geqslant 2$. We identify $\Delta_{0}=\mathbb{Z} / n \mathbb{Z}$ with $\{1,2, \ldots, n\}$; given an integer $z$, its residue class modulo $n$ will be denoted by $\bar{z}$, and thus $1 \leqslant \bar{z} \leqslant n$.

4.1. Adding and deleting parts. If $l=\left(l_{1}, \ldots, l_{n}\right)$ is a partition, then $l^{\prime}=$ $\left(l_{1}, \ldots, l_{i-1}, l_{i+1}, \ldots, l_{n}\right)$ is said to be obtained from $l$ by deleting a part of length $l_{i}$, and $l$ is said to be obtained from $l^{\prime}$ by adding a part of length $l_{i}$.

Separated $n$-tuples of partitions. We will consider $n$-tuples $\pi=\left(\pi^{(1)}, \ldots, \pi^{(n)}\right)$ of partitions. The height of an $n$-tuple $\pi$ is, by definition, the maximum of the heights of $\pi^{(i)}$, for $1 \leqslant i \leqslant n$. We recall from the introduction that an $n$-tuple $\pi=\left(\pi^{(1)}, \ldots, \pi^{(n)}\right)$ will be called separated provided that, for every $t \geqslant 1$, there is some $1 \leqslant a(t) \leqslant n$ such that $\tilde{\pi}^{(a(t))}$ has no part of length $t$, and that $\Pi^{s}$ will denote the set of separated $n$-tuples of partitions. This set $\Pi^{s}$ will be our basic index set. Given a (separated) $n$-tuple $\pi=\left(\pi^{(1)}, \ldots, \pi^{(n)}\right)$ of partitions of length $m$, a function $a:\{1, \ldots, m\} \rightarrow \mathbb{Z} / n \mathbb{Z}$ such that $\tilde{\pi}^{(a(t))}$ has no part of length $t$ will be called a separator for $\pi$. The set of pairs $(\pi, a)$, where $\pi$ is an $n$-tuple of partitions and $a$ is a separator for $\pi$, will be denoted by $\Pi^{s s}$. There is a unique pair $(\pi, a)$ with $\pi=((0), \ldots,(0))$, called the empty pair. Our convention of identifying $\mathbb{Z} / n \mathbb{Z}$ with $\{1,2, \ldots, n\}$ provides a minimal separator $a_{\pi}$, for any $\pi \in \Pi^{s}$ (with $a_{\pi}(t)$ defined as follows: $\pi^{\left(a_{\pi}(t)\right)}$ has no part of length $t$, whereas $\pi^{(i)}$ has a part of length $t$, for all $\left.1 \leqslant i<a_{\pi}(t)\right)$.

4.2. Towers of periodic functions. A tower $\varphi=(\varphi(1), \ldots, \varphi(h))$ of periodic functions is given by a chain $I_{1} \subset I_{2} \subset \ldots \subset I_{h}$, where $I_{t}$ is a set of $t(n-1)$ consecutive integers, and $\varphi(t): I_{t} \rightarrow \mathbb{N}_{0}$ is an $(n-1)$-periodic function (the image of $i \in I_{t}$ under $\varphi(t)$ will be denoted by $\varphi(t)_{i}$, the periodicity amounts to the equalities $\varphi(t)_{i+n-1}=\varphi(t)_{i}$, for $\left.i, i+n-1 \in I_{t}\right)$. A tower $\varphi$ of periodic functions will be said to be normalized provided either it is the zero tower, or else $\varphi(h) \neq 0$ and at least one of 0 and 1 belongs to $I_{1}$. We denote by $\Phi$ the set of normalized towers of periodic functions. (The reason for the last normalization condition is the following: towers of periodic functions which are obtained from each other by shifting the intervals $I_{t}$ by a fixed multiple of $n$ will behave rather similarly; using such a shift, we may always assume that 0 or 1 belongs to $I_{1}$.)

Given a tower $\varphi=(\varphi(1), \ldots, \varphi(h))$ of periodic functions with $\varphi(t): I_{t} \rightarrow \mathbb{N}_{0}$, we define $\hat{\varepsilon}(\varphi) \in \Pi^{s s}$ as follows: when $\varphi$ is the zero tower, let $\hat{\varepsilon}(\varphi)$ be the empty pair. Otherwise, let $I_{t}=\{a(t)-1, \ldots, a(t)-t(n-1)\}$, for $1 \leqslant t \leqslant h$, and define partitions $\pi^{(1)}, \ldots, \pi^{(n)}$ so that, for $1 \leqslant i \leqslant n-1$, the partition $\tilde{\pi}^{(\overline{a(t)}-i)}$ has precisely $\varphi(t)_{a-i}$ parts of length $t$, whereas $\tilde{\pi}^{(\overline{a(t)})}$ has no part of length $t$. By definition, the function $\bar{a}$ (with $\bar{a}(t)=\overline{a(t)}$ ) is a separator for $\left(\pi^{(1)}, \ldots, \pi^{(n)}\right)$; thus $\hat{\varepsilon}(\varphi):=(\pi, \bar{a})$ belongs to $\Pi^{s s}$ (and we let $\varepsilon(\varphi):=\pi$ ).

\section{Lemma. The map $\hat{\varepsilon}: \Phi \rightarrow \Pi^{s s}$ is bijective.}

Proof. The inverse map is constructed as follows: let $\pi$ be a separated $n$-tuple of partitions, and let $a$ be a separator for $\pi$. Let $h$ be the height of $\pi$, and assume $h \geqslant 1$. We define inductively $a^{\prime}(t) \in \mathbb{Z}$ as follows: $a^{\prime}(1)=a(1)$, and, for $t \geqslant 2$, let $a^{\prime}(t)$ be the smallest number with $a^{\prime}(t) \equiv a(t)(\bmod n)$ and $a^{\prime}(t-1) \leqslant a^{\prime}(t)$. Let $I_{t}=\left\{z \in \mathbb{Z} \mid a^{\prime}(t)-1 \leqslant z \leqslant a^{\prime}(t)-t(n-1)\right\}$. Since $0 \leqslant a^{\prime}(t)-a^{\prime}(t-1) \leqslant n-1$, it 
follows that $I_{t-1} \subseteq I_{t}$, for all $2 \leqslant t \leqslant n$. It remains to define $\varphi(t): I_{t} \rightarrow \mathbb{N}_{0}$, for all $1 \leqslant t \leqslant h$. Since we want to have that $\varphi(t)$ is $(n-1)$-periodic, it suffices to define $\varphi(t)_{a^{\prime}(t)-i}$ for $1 \leqslant i \leqslant n-1$, and extend $\varphi(t)$ periodically. For $1 \leqslant i \leqslant n-1$, let $\varphi(t)_{a^{\prime}(t)-i}$ be the number of parts of $\tilde{\pi}^{\left(a^{\prime}(t)-i\right)}$ of length $t$. In this way, we obtain a tower $\varphi$ of periodic functions, and clearly $\varphi$ is normalized.

4.3. Words. Let us consider $\Omega=\Omega(\mathbb{Z} / n \mathbb{Z})$. The words in $\Omega$ of the form $a[l]=a(\overline{a+1}) \ldots(\overline{a+l-1})$ for some $a \in \mathbb{Z}$ and some $l \in \mathbb{N}_{1}$ will be said to be indecomposable. By definition, $a[0]=1$, for all $1 \leqslant a \leqslant n$, but this is not an indecomposable word.

Any word $w \neq 1$ in $\Omega=\Omega(\mathbb{Z} / n \mathbb{Z})$ can be written in scooping form,

$$
w=(\overline{z-1})^{e_{z-1}}(\overline{z-2})^{e_{z-2}} \ldots(\overline{z-m})^{e_{z-m}},
$$

where $z \in \mathbb{Z}, e_{z-t} \geqslant 0$ for all $1 \leqslant t \leqslant m$ and $m$ minimal (the minimality of $m$ is equivalent to the requirements $e_{z-1} \geqslant 1, e_{z-m} \geqslant 1$, and that there are no $n-1$ consecutive zeros in the sequence $\left.\left(e_{z-1}, e_{z-2}, \ldots, e_{z-m}\right)\right)$. Note that for $w$ in scooping form $(*)$, the data $\overline{z-1}$ and $e_{z-1}, e_{z-2}, \ldots, e_{z-m}$ are uniquely determined by $w$. We say that $w$ is of (scooping) height $h$, provided $h=\lceil m /(n-1)\rceil$, where $\lceil x\rceil$ is the smallest integer $y$ satisfying $x \leqslant y$. By definition, the scooping height of 1 is 0 .

Lemma. Let $w$ be a word of scooping height $h$, and $v$ an indecomposable subword of $w$. Then the length of $v$ is at most $h$, and if the length of $v$ is $h$, then $v$ has a unique complementary subword in $w$.

Proof. Let (*) be the scooping form of $w$. Let $v=a[l]$ for some $1 \leqslant a \leqslant n$ and some $l \in \mathbb{N}_{1}$, and assume $v=w \mid I$, where $I=\left\{i_{1}<i_{2}<\ldots<i_{l}\right\}$. Assume that $l \geqslant h$. Consider $w^{\prime}=(\overline{z-n})^{e_{z-n}}(\overline{z-n-1})^{e_{z-n-1}} \ldots(\overline{z-m})^{e_{z-m}}$; this is a word of scooping height at most $h-1$, and thus by induction, $v$ cannot be a subword of $w^{\prime}$. It follows that $a \equiv z-t(\bmod n)$ for some $1 \leqslant t \leqslant n-1$, and $\sum_{i=1}^{t-1} e_{z-i}<i_{1} \leqslant$ $\sum_{i=1}^{t} e_{z-i}$. The second letter of $a[l]$ is $\overline{a+1}=\overline{z-t+1}$, and this is the $i_{2}$ th letter of $w$. But $\overline{z-t+1}$ does not occur in $(\overline{z-t})^{e_{z-1}}(\overline{z-t+1})^{e_{z-t+1}} \ldots(\overline{z-n+1})^{e_{z-n+1}}$; thus $\sum_{i=1}^{n-1} e_{z-i}<i_{2}$, and $v^{\prime}=(\overline{a+1})[l-1]$ is a subword of $w^{\prime}$. By induction, $l-1 \leqslant$ $h-1$, and thus $l=h$. Also by induction, the complementary subword $u^{\prime}$ of $v^{\prime}$ in $\boldsymbol{w}^{\prime}$ is uniquely determined; thus there is only one complementary subword $u$ for $v$ in $w$, namely, $(z-1)^{e_{z-1}^{\prime}} \ldots(z-n+1)^{e_{-}^{\prime}-n+1} u^{\prime}$, where $e_{z-i}^{\prime}=e_{z-i}$ for all $i \neq t$, and $e_{z-t}^{\prime}=e_{z-t}-1$.

4.4. Condensed words. Let $\varphi=(\varphi(1), \ldots, \varphi(h))$ be a tower of periodic functions, with $\varphi(t): I_{t} \rightarrow \mathbb{N}_{0}$, for $1 \leqslant t \leqslant h$. We extend $\varphi(t)$ to all of $I_{h}$ by zero; thus $\varphi(t)_{i}=0$ for $i \in I_{h} \backslash I_{t}$. Let $e=e(\varphi): I_{h} \rightarrow \mathbb{N}_{0}$ be defined by $e_{i}=\sum_{t=1}^{h} \varphi(t)_{i}$. Note that the function $e(\varphi): I_{h} \rightarrow \mathbb{N}_{0}$ together with the subsets $I_{t}$, for $1 \leqslant t<h$, determine $\varphi$ uniquely. We define $\omega(\varphi) \in \Omega$ by

$$
\left.\omega(\varphi)=(\overline{z-1})^{e_{z-1}}(\overline{z-2})^{e_{z-2}} \ldots(\overline{z-h(n-1})\right)^{e_{z-h(n-1)}},
$$

where $I_{h}=\{z-1, z-2, \ldots, z-h(n-1)\}$. The words $\omega(\varphi)$ obtained in this way will be called condensed words. In particular, 1 is a condensed word, since $1=\omega(\varphi)$ for $\varphi$ the empty tower. We denote by $\Omega^{c}$ the set of condensed words. (Clearly, for forming condensed words, we may assume that we start with a 
normalized tower of periodic functions: if $\varphi$ is not the zero tower, we may assume $\varphi(h) \neq 0$, and we may shift the intervals $I_{t}$ by a fixed multiple of $n$, since the letters of $\omega(\varphi)$ are residue classes modulo $n$ ).

Remark. If $\varphi(h) \neq 0$, then there are at most $n-2$ consecutive zeros in the sequence $\left(e_{z-1}, e_{z-2}, \ldots, e_{z-h(n-1)}\right)$; also, if $i$ is minimal with $e_{z-i} \neq 0$, and $j$ is maximal with $e_{z-j} \neq 0$, then $(h-1)(n-1)<j-i+1 \leqslant h(n-1)$. It follows that the scooping form of $\omega(\varphi)$ is

$$
\omega(\varphi)=(\overline{z-i})^{e_{z-i}}(\overline{z-i-1})^{e_{z-i-1}} \ldots(\overline{z-j})^{e_{z-i}},
$$

and the scooping height of $\omega(\varphi)$ is $h$.

ExAMPLE. Let $n=4$. Let

$$
\begin{aligned}
& I_{1}=\{-1,0,1\}, \quad I_{2}=\{-3,-2, \ldots, 2\}, \quad I_{3}=\{-4,-3, \ldots, 4\}, \\
& I_{4}=\{-4,-3, \ldots, 7\}, \quad I_{5}=\{-4,-3, \ldots, 10\} .
\end{aligned}
$$

Let $\varphi(t)$ be defined by the initial values

$$
\begin{array}{lll}
\varphi(1)_{-1}=1, & \varphi(1)_{0}=0, & \varphi(1)_{1}=2, \\
\varphi(2)_{-3}=0, & \varphi(2)_{-2}=2, & \varphi(2)_{-1}=0, \\
\varphi(3)_{-4}=1, & \varphi(3)_{-3}=1, & \varphi(3)_{-2}=1, \\
\varphi(4)_{-4}=0, & \varphi(4)_{-3}=0, & \varphi(4)_{-2}=0, \\
\varphi(5)_{-4}=0, & \varphi(5)_{-3}=1, & \varphi(5)_{-2}=0 .
\end{array}
$$

Then $z=11$, and the values of $z-i$ and $e_{z-i}$, for $1 \leqslant i \leqslant 15=h(n-1)$, are given as follows:

\begin{tabular}{l|rrrrrrrrrrrrrrr}
$z-i$ & 10 & 9 & 8 & 7 & 6 & 5 & 4 & 3 & 2 & 1 & 0 & -1 & -2 & -3 & -4 \\
$\overline{\overline{z-i}}$ & 2 & 1 & 4 & 3 & 2 & 1 & 4 & 3 & 2 & 1 & 4 & 3 & 2 & 1 & 4 \\
\hline$e_{z-i}$ & 0 & 1 & 0 & 0 & 1 & 0 & 1 & 2 & 1 & 5 & 2 & 2 & 3 & 2 & 1
\end{tabular}

Thus $\omega(\varphi)=1243^{2} 21^{5} 4^{2} 3^{2} 2^{3} 1^{2} 4$.

Lemma. Let $w \neq 1$. If $w$ is condensed, then there exists an indecomposable subword $v$ of $w$ with length of $v$ equal to the scooping height of $w$, and for any such $v$, the complementary subword is condensed.

Proof. Let $w=\omega(\varphi)$ for some normalized tower $\varphi=(\varphi(1), \ldots, \varphi(h))$ of periodic functions; where $\varphi(t): I_{t} \rightarrow \mathbb{N}_{0}$. Let $I_{h}=\{z-1, \ldots, z-t(n-1)\}$; thus $w=(\overline{z-1})^{e_{z-1}} \ldots(\overline{z-h(n-1)})^{e_{z-h(n-1)}}$. We see that the scooping height of $w$ is at most $h$. Since $\varphi(h) \neq 0$, there is some $1 \leqslant t \leqslant n-1$ such that $\varphi(h)_{t} \neq 0$. Then $(\overline{z-h})[h]$ is a subword of $\omega(\varphi)$, it is indecomposable, and of length $h$; thus the scooping height of $w$ has to be $h$. The complementary subword will be $\omega\left(\varphi^{\prime}\right)$, where $\varphi^{\prime}=\left(\varphi(1), \ldots, \varphi(h-1), \varphi^{\prime}(h)\right)$ with $\varphi^{\prime}(h)_{i}=\varphi(h)_{i}-1$ for all $i$ with $i \equiv t(\bmod n-1)$, and $\varphi^{\prime}(h)_{i}=\varphi(h)_{i}$ for all $i$ with $i \not \equiv t(\bmod n-1)$.

Remark. For $n=2$, the word $w=1^{2} 212^{2}$ is of scooping height 4 , the subword $v=1212$ is indecomposable and of length 4, and the complementary word $u$ for $v$ 
in $w$ is $u=12$. Note that $u$ is condensed, whereas $w$ is not condensed. This shows that the converse of the lemma is not valid.

The lemma yields a direct way of associating to a condensed word $w$ an $n$-tuple $\varepsilon(w)$ of partitions: for $w=1$, let $\varepsilon(1)=((0), \ldots,(0))$. For $w$ a condensed word of scooping height $h \geqslant 1$, choose an indecomposable subword $v$ of $w$ of length $h$, say $v=a[h]$ where $1 \leqslant a \leqslant n$, and let $u$ be the complementary subword for $v$ in $w$. Inductively, $\varepsilon(u)$ is defined, and $\varepsilon(w)$ is obtained by adding to the partition $\varepsilon(u)^{(a)}$ a part of length $h$, and keeping the remaining $\varepsilon(u)^{(i)}$. Note that for $\varphi \in \Phi$, we have

$$
\varepsilon(\omega(\varphi))=\varepsilon(\varphi)
$$

Altogether, we have the following commutative diagram:

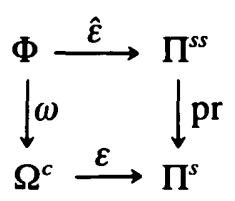

where pr: $\Pi^{s s} \rightarrow \Pi^{s}$ is the canonical projection, $\operatorname{pr}(\pi, a)=\pi$, for $(\pi, a) \in \Pi^{s s}$.

Definition of the word $\omega_{\pi}$, for $\pi \in \Pi^{s}$. For $\pi \in \Pi^{s}$, we define

$$
\omega_{\pi}=\omega \hat{\varepsilon}^{-1}\left(\pi, a_{\pi}\right)
$$

here, we use the minimal separator $a_{\pi}$ as defined in $\S 4.1$, and the fact that $\hat{\varepsilon}: \Phi \rightarrow \Pi^{s s}$ is bijective. In this way, we obtain for $\pi \in \Pi^{s}$ an explicit word $\omega_{\pi}$ such that $\varepsilon\left(\omega_{\pi}\right)=\pi$. Actually, in all our considerations, we may and will consider, instead of $\omega_{\pi}$, any condensed word $w_{\pi}$ such that $\varepsilon\left(w_{\pi}\right)=\pi$.

4.5. Dimension vectors. For any $n$-tuple $\pi$ of partitions, for any tower $\varphi$ of periodic functions, as well as for any word $w \in \Omega=\Omega(\mathbb{Z} / n \mathbb{Z})$, we are going to define its dimension vector $\operatorname{dim} \pi, \operatorname{dim} \varphi$, and $\operatorname{dim} w$, respectively, the dimension vectors being $n$-tuples of non-negative integers. Let $1 \leqslant j \leqslant n$; we define the $j$ th component of the dimension vector as follows. Let $\pi$ be an $n$-tuple of partitions. Then

$$
(\operatorname{dim} \pi)_{j}=\sum \pi_{i}^{(a)},
$$

where the summation extends over all pairs $(a, i)$ satisfying $\overline{a+i-1}=j$. We will give below a visualization of these numbers $(\operatorname{dim} \pi)_{j}$. For pairs $(\pi, a) \in \Pi^{s s}$, let $\operatorname{dim}(\pi, a)=\operatorname{dim} \pi$.

Let $\varphi$ be a tower of periodic functions. Then, by definition,

$$
(\operatorname{dim} \varphi)_{j}=\sum_{t \geqslant 1} \sum_{i=j} \varphi(t)_{i}
$$

Let $w \in \Omega$, say $w=s_{1}, \ldots, s_{n}$ with $1 \leqslant s_{i} \leqslant n$ for all $i$. Then we define $(\operatorname{dim} w)_{j}$ as the number of indices $i$ such that $s_{i}=j$.

Lemma. The maps $\hat{\varepsilon}: \Phi \rightarrow \Pi^{s s}, \varepsilon: \Omega^{c} \rightarrow \Pi^{s}, \omega: \Phi \rightarrow \Omega^{c}$ and $\mathrm{pr}: \Pi^{s s} \rightarrow \Pi^{s}$ commute with dim. 
The proof is straightforward.

Given $\mathbf{d} \in \mathbb{N}_{0}^{n}$, we denote by $\Pi_{d}^{s}$ the set of separated $n$-tuples $\pi$ of partitions, with $\operatorname{dim} \pi=\mathbf{d}$.

4.6. The type of a column. Consider a fixed column, say the squares $(a, 1, j),(a, 2, j), \ldots,(a, h, j)$ with $a, j$ fixed, and $h=\tilde{\pi}_{j}^{(a)}$. The square $(a, 1, j)$ will be called the top of the column, the square $(a, h, j)$ the socle of the column.

The sequence of types $s(a, 1, j), s(a, 2, j), \ldots, s(a, h, j)$ yields the word

$$
s(a, 1, j) s(a, 2, j) \ldots s(a, h, j)=a[h] ;
$$

thus we will say that this column is of type $a[h]$. In this way, we may speak of adding or removing columns: if $\pi=\left(\pi^{(1)}, \ldots, \pi^{(n)}\right), \lambda=\left(\lambda^{(1)}, \ldots, \lambda^{(n)}\right)$, with $\pi^{(i)}=\lambda^{(i)}$ for all $i \neq a$, and $\tilde{\lambda}^{(a)}$ is obtained from $\tilde{\pi}^{(a)}$ by deleting a part of length $h$, then we will say that $\lambda$ is obtained from $\pi$ by deleting a column of type $a[h]$, and $\pi$ is obtained from $\lambda$ by adding a column of type $a[h]$. (Note that we allow the case $h=0$, which amounts to $\pi=\lambda$.)

4.7. The natural ordering on $\Pi$. We are going to introduce a partial ordering on the set $\Pi$ of $n$-tuples of partitions. Let $\pi, \lambda \in \Pi$. We write $\lambda \leqslant \pi$ provided there are integers $r \geqslant 1, s \geqslant 0, t \geqslant 1$, and $1 \leqslant a \leqslant n$ such that the $n$-tuple $\mu \in \Pi$ obtained from $\pi$ by deleting one column of type $a[r+s+t]$, and one column of type $\overline{a+r}[s]$, is the same as that obtained from $\lambda$ by deleting one column of type $a[r+s]$ and another column of type $\overline{a+r}[s+t]$ (note that for obtaining $\mu$ from $\lambda$, we have to stress that we remove two columns, since we allow $r \equiv 0(\bmod n)$ and $r=t$ ). We write $\lambda \leqslant \pi$ in the case where there is a sequence $\lambda=\lambda_{0} \leqslant \lambda_{1} \leqslant \ldots \leqslant$ $\lambda_{m}=\pi$ of length $m \geqslant 0$. It is easy to see that $\leqslant$ is a partial ordering, and we call this the natural ordering on $\Pi$.

\section{Lemma. Let $\lambda \leqslant \pi$ in $\Pi$. Then $\operatorname{dim} \lambda=\operatorname{dim} \pi$.}

Proof. We may assume $\lambda \leqslant \pi$. In order to obtain $\mu$, we delete from $\pi$ columns of type $a[r+s+t]$ and $\overline{a+r}[s]$, and to obtain $\lambda$, we add to $\mu$ columns of type $a[r+s]$ and $\overline{a+r}[s+t]$. The squares of $\mu$ do not change their type when considered as squares of $\pi$ or of $\lambda$. On the other hand, we clearly have

$$
\operatorname{dim} a[r+s+t]+\operatorname{dim} \overline{a+r}[s]=\operatorname{dim} a[r+s]+\operatorname{dim} \overline{a+r}[s+t] .
$$

\section{Tableaux}

5.1. THEOREM 1. Let $w$ be a condensed word, and $\pi$ an $n$-tuple of partitions. Then the following assertions are equivalent:

(i) there exists a column-increasing $\pi$-tableau $T$ with $\omega(T)=w$;

(ii) there exists a standard $\pi$-tableau $T$ with $\omega(T)=w$;

(iii) $\pi \leqslant \varepsilon(w)$.

The proof will be given in this section. We need some preparations. First, consider an individual partition $l$ (note that we also use the definitions exhibited above in the case $n=1$ ). 
5.2. Reordering Lemma. Let $T$ be a column-increasing l-tableau. Then there exists a permutation $\sigma$ of $\|l\|$ fixing the row-index such that $T \circ \sigma$ is a standard l-tableau.

Proof. First, let $T$ be an arbitrary $l$-tableau. Fix some $i$ and consider the values $T(i, j)$ with $1 \leqslant j \leqslant l_{i}$. There is a permutation $\sigma_{i}$ of $\left\{1,2, \ldots, l_{i}\right\}$ such that

$$
T\left(i, \sigma_{i}(1)\right)<T\left(i, \sigma_{i}(2)\right)<\ldots<T\left(i, \sigma_{i}\left(l_{i}\right)\right) .
$$

Let $\sigma(i, j)=\left(i, \sigma_{i}(j)\right)$; thus $\sigma$ is a permutation of $\|\pi\|$ fixing the row-index, and, by construction, $T \circ \sigma$ is row-increasing. Assume that $T$ is column-increasing. Let $(i, j),(i+1, j) \in\|\pi\|$. Now, $(T \circ \sigma)(i, j)$ is the maximum of the values

$$
(T \circ \sigma)(i, t)=T\left(i, \sigma_{i}(t)\right),
$$

with $1 \leqslant t \leqslant j$, and these are the $j$ smallest possible values. Thus this maximum is less than or equal to the maximum over $j$ arbitrary values of $T$ on the $i$ th row; and we take the squares $\left(i, \sigma_{i+1}(t)\right)$ with $1 \leqslant t \leqslant j$ :

$$
(T \circ \sigma)(i, j)=\max _{1 \leqslant i \leqslant j} T\left(i, \sigma_{i}(t)\right) \leqslant \max _{1 \leqslant t \leqslant j} T\left(i, \sigma_{i+1}(t)\right) .
$$

But $T\left(i, \sigma_{i+1}(t)\right)<T\left(i+1, \sigma_{i+1}(t)\right)$, since $T$ is column-increasing; therefore

$$
\max _{1 \leqslant t \leqslant j} T\left(i, \sigma_{i+1}(t)\right)<\max _{1 \leqslant t \leqslant j} T\left(i+1, \sigma_{i+1}(t)\right)=(T \circ \sigma)(i+1, j) .
$$

This shows that $(T \circ \sigma)(i, j)<(T \circ \sigma)(i+1, j)$.

Remark. Let $\pi$ be an $n$-tuple of partitions, and $T$ a $\pi$-tableau. Let $\sigma$ be a permutation of $\|\pi\|$ fixing the partition-index and the row-index. Then

$$
s \circ(T \circ \sigma)^{-1}=s \circ T^{-1},
$$

and thus $\omega(T)=\omega(T \circ \sigma)$.

Proof. Let $(a, i, j) \in\|\pi\|$ and let $\sigma(a, i, j)=\left(a, i, j^{\prime}\right) ;$ thus $u:=(T \circ \sigma)(a, i, j)=$ $T\left(a, i, j^{\prime}\right)$. Then $s \circ(T \circ \sigma)^{-1}(u)=s(a, i, j)=\overline{a+i-1}=s\left(a, i, j^{\prime}\right)=(s \circ T)(u)$.

COROLLARY. If $T$ is a column-increasing $\pi$-tableau, then reordering of the rows of $\|\pi\|$ yields a standard $\pi$-tableau $T^{\prime}$ with $\omega\left(T^{\prime}\right)=\omega(T)$.

In particular, we obtain in this way the equivalence of (i) and (ii) in Theorem 1.

5.3. Let $c$ be a tower of periodic functions. We are going to construct a standard $\varepsilon(\varphi)$-tableau $T_{\varphi}$ as follows.

Let $\varphi=(\varphi(1), \ldots, \varphi(h))$ with $\varphi(h) \neq 0$, let $I_{h}=\{z-1, \ldots, z-h(n-1)\}$. There is $z-1 \leqslant a \leqslant z-n+1$ with $\varphi(h)_{a} \neq 0$. For $1 \leqslant t \leqslant h$, let

$$
a_{t}=a+(t-1)(n-1) \text {. }
$$

As usual, $e=e(\varphi)$, and we denote $u_{t}=\sum_{i>a_{t}} e_{i}$. Since $\varphi(h)_{a} \geqslant 1$, the $(n-1)$ periodicity of $\varphi(h)$ yields $\varphi(h)_{a_{t}} \geqslant 1$ for all $1 \leqslant t \leqslant h$. Let $\omega(\varphi)=s_{1} s_{2} \ldots s_{n}$, with $s_{i} \in \mathbb{Z} / n \mathbb{Z}$, and we note that $s_{u_{t}+1}=\bar{a}_{t}$. The first column of $\varepsilon(\varphi)^{(\bar{a})}$ has length $h$, and we define $T_{\varphi}(\bar{a}, t, 1)=u_{t}+1$, for $1 \leqslant t \leqslant h$.

The remaining squares of $\|\varepsilon(\varphi)\|$ may be considered as the squares of $\left\|\varepsilon\left(\varphi^{\prime}\right)\right\|$, where $\varphi^{\prime}=\left(\varphi(1), \varphi(2), \ldots, \varphi(h-1), \varphi^{\prime}(h)\right)$, and $\varphi^{\prime}(h)_{i}=\varphi(h)_{i}-1$ for $i \equiv a$ 
$(\bmod n-1)$, and $\varphi^{\prime}(h)_{i}=\varphi(h)_{i}$ for $i \not \equiv a(\bmod n-1)$. By induction, $T_{\varphi^{\prime}}$ is defined. Let

$$
\iota:\{1,2, \ldots,|\pi|-h\} \rightarrow\{1,2, \ldots,|\pi|\} \backslash\left\{u_{t}+1 \mid 1 \leqslant t \leqslant h\right\}
$$

be monoton and bijective, and define

$$
T_{\varphi}(b, i, j)= \begin{cases}\iota T_{\varphi^{\prime}}(b, i, j) & \text { for } b \neq \bar{a}, \\ \iota T_{\varphi^{\prime}}(b, i, j-1) & \text { for } b=\bar{a}, j>1, \\ u_{t}+1 & \text { for } b=\bar{a}, j=1\end{cases}
$$

It is obvious that $T_{\varphi}$ is column-increasing: by induction, this is true for $T_{\varphi^{\prime}}$, and for $1 \leqslant t<h$, we have $u_{t}<u_{t+1}$; thus $T_{\varphi}$ also increases on the first column of $\|\varepsilon(\varphi)\|$. Similarly, by induction, $T_{\varphi^{\prime}}$ is row-increasing on $\varepsilon\left(\varphi^{\prime}\right)$. It is not hard to see that for a square $(\bar{a}, t, 2)$ in $\|\varepsilon(\varphi)\|$, we have $u_{t}+1<T(\bar{a}, t, 2)$. Therefore, $T_{\varphi}$ is also row-increasing.

Remark. Avoiding induction, we may write down $\varepsilon(\varphi)$, and $T_{\varphi}$ directly, as follows. Let $I_{t}=\{a(t)-1, \ldots, a(t)-t(n-1)\}$. For $a(t)-1 \leqslant a \leqslant a(t)-n+1$, the partition $\varepsilon(\varphi)^{(\vec{a})}$ will have precisely $\varphi(t)_{a}$ columns of length $t$. Assume that these columns have column-index $j+1, j+2, \ldots, j+\varphi(t)_{a}$. Then, for $1 \leqslant s \leqslant \varphi(t)_{a}$,

$$
T(\bar{a}, i, j+s)=s+\sum_{i>a+(i-1)(n-1)} e_{i}+\sum_{i^{\prime}>t} \varphi\left(t^{\prime}\right)_{a+(i-1)(n-1)} .
$$

The tower $\varphi$ presented as the example in $\S 4.4$ yields the $\varepsilon(\varphi)$-tableau shown in Fig. 1.
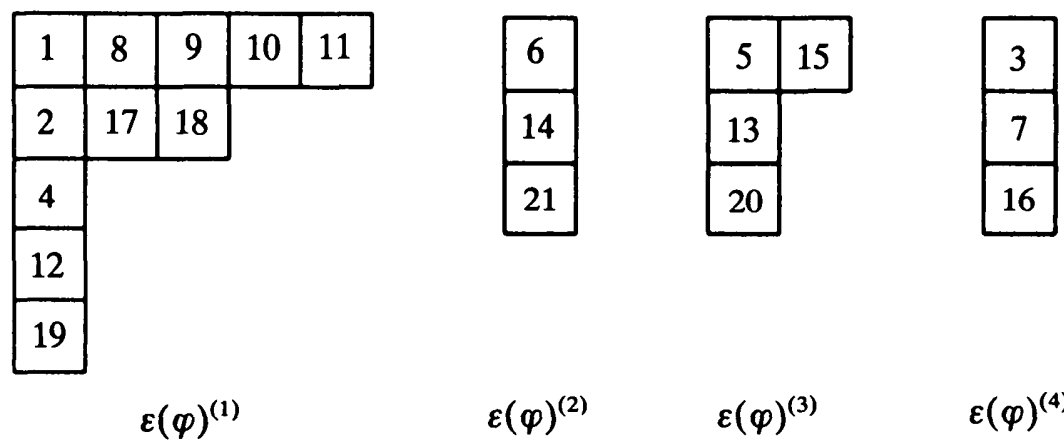

$$
\varepsilon(\varphi)^{(1)} \quad \varepsilon(\varphi)^{(2)} \quad \varepsilon(\varphi)^{(3)} \quad \varepsilon(\varphi)^{(4)}
$$

Fig. 1

There is an effective way for writing down $\varepsilon(\varphi)$ and $T_{\varphi}$ simultaneously. Display the functions $\varphi(t): I_{t} \rightarrow \mathbb{N}_{0}$ by a bar diagram, with $I_{t}$ arranged vertically, and $\mathbb{N}_{0}$ horizontally, starting with $\varphi(h)$ on the left, and continuing to the right until we reach the bar diagram of $\varphi(1)$. Now number the squares line by line, and only afterwards separate the squares into columns and distribute them to form the partitions $\varepsilon(\varphi)^{(1)}, \ldots, \varepsilon(\varphi)^{(n)}$. In our example, the sequence of bar diagrams together with the numbering looks as shown in Fig. 2; we have added a circle to the top squares of the columns which arise. 


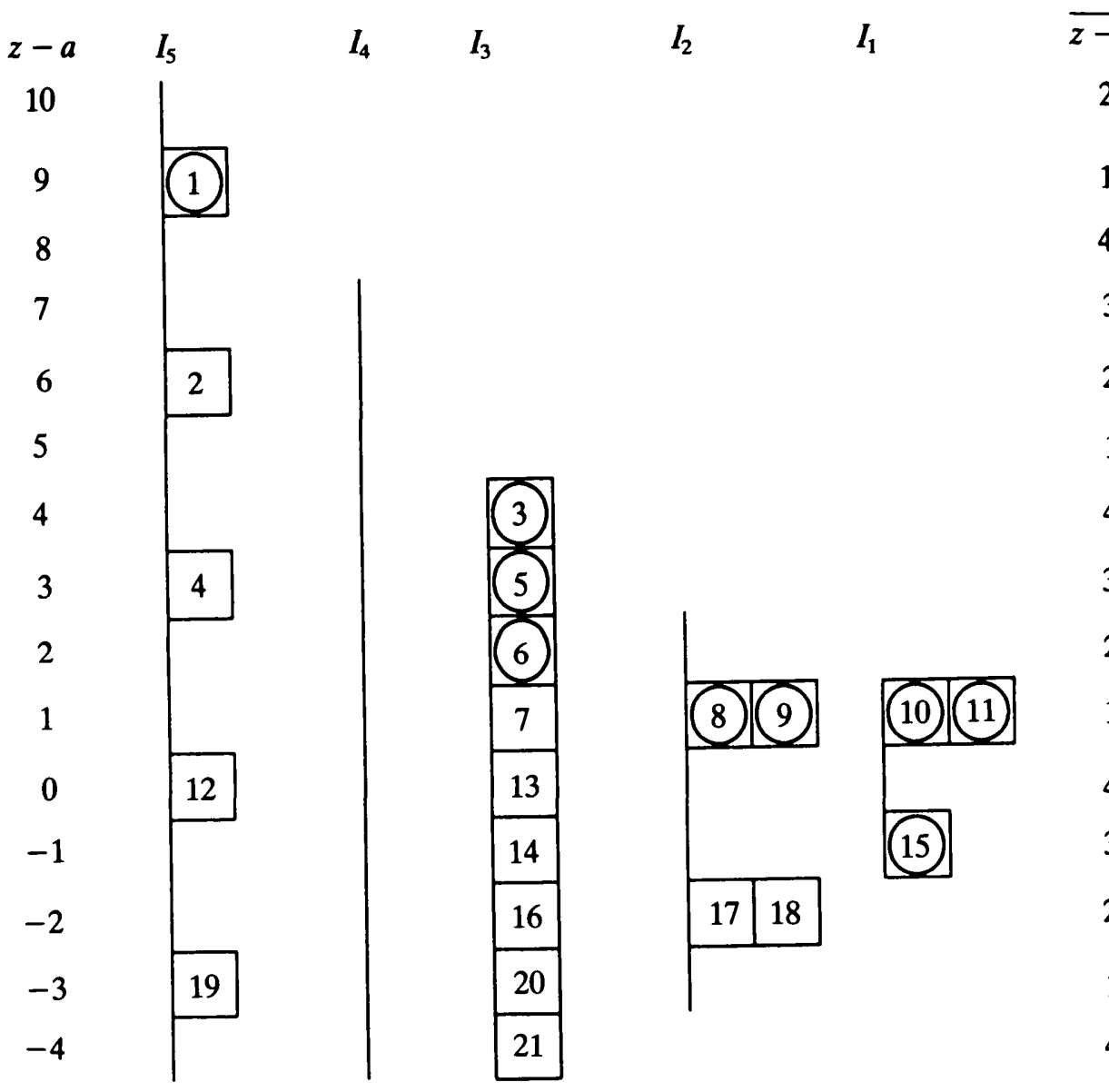

FIG. 2

5.4. Lemma. Let $\lambda \leqslant \pi$ in $\Pi$. Let $T$ be a column-increasing $\pi$-tableau. Then there exists a column-increasing $\lambda$-tableau $T^{\prime}$ with $\omega\left(T^{\prime}\right)=\omega(T)$.

If $T$ is a $\pi$-tableau, it sometimes will be convenient to abuse the notation as follows: let $1 \leqslant a \leqslant n, 1 \leqslant j \leqslant \pi_{1}^{(a)}$. Then we define

$$
T(a, 0, j)=0 \quad \text { and } \quad T\left(a, \tilde{\pi}_{j}^{(a)}+1, j\right)=|\pi|+1 .
$$

Proof. We only have to consider the case $\lambda \leqslant \pi$. So assume that there are integers $r \geqslant 1, s \geqslant 0, t \geqslant 1$ and $1 \leqslant a \leqslant n$ such that we obtain the same $\mu \in \Pi$ when deleting one column of type $a[r+s+t]$ and one of type $\overline{a+r}[s]$ from $\pi$, and when deleting one column of type $a[r+s]$ and one of type $\overline{a+r}[s+t]$ from $\lambda$. Let $T$ be a column-increasing $\pi$-tableau; we are going to define a corresponding $\lambda$-tableau $T^{\prime}$. By definition, $T^{\prime}$ shall coincide with $T$ on the columns which are not changed. (Note that this is easy to say and easy to visualize, but our notation is not well-adjusted for writing this down with formulae: deletion of columns and 
addition of columns will always change the column index of the remaining columns!)

Assume we have removed from $\pi^{(a)}$ the $j$ th column, with squares $(a, 1, j),(a, 2, j), \ldots,(a, r+s+t, j)$, and from $\pi^{(a+r)}$ the $j^{\prime}$ th column, with squares $\left(\overline{a+r}, 1, j^{\prime}\right),\left(\overline{a+r}, 2, j^{\prime}\right), \ldots,\left(\overline{a+r}, s, j^{\prime}\right)$. Let $u_{i}=T(a, i, j)$, for $1 \leqslant i \leqslant r+s+t$ and $v_{i}=T\left(\overline{a+r}, i, j^{\prime}\right)$, for $0 \leqslant i \leqslant s+1$. Then, we deal with two columns of the shape illustrated in Fig. 3, and we have inserted into the squares the values under $T$ and squares on the same horizontal level have the same type.

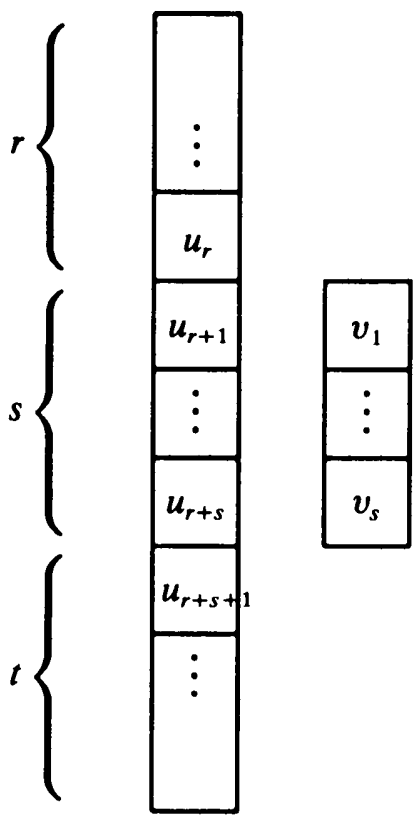

Fig. 3

Now consider $\lambda$, and assume we have removed from $\lambda^{(a)}$ the $k$ th column with squares $(a, 1, k),(a, 2, k), \ldots,(a, r+s, k)$ and from $\lambda^{(\overline{a+r})}$ the $k^{\prime}$ th column with squares $\left(\overline{a+r}, 1, k^{\prime}\right), \ldots,\left(\overline{a+r}, 2, k^{\prime}\right), \ldots,\left(\overline{a+r}, s+t, k^{\prime}\right)$, in order to obtain $\mu$. We have to assign to these squares the values $u_{i}, v_{i}$. Suppose there is some $0 \leqslant i_{0} \leqslant s$ with both

Then let

$$
u_{r+i_{0}}<v_{i_{0}+1} \text { and } v_{i_{0}}<u_{r+i_{0}+1}
$$

$$
\begin{aligned}
T^{\prime}(a, i, k) & = \begin{cases}u_{i} & \text { for } 1 \leqslant i \leqslant r+i_{0}, \\
v_{i-r} & \text { for } r+i_{0}<i \leqslant r+s,\end{cases} \\
T^{\prime}\left(\overline{a+r}, i, k^{\prime}\right) & = \begin{cases}v_{i} & \text { for } 1 \leqslant i \leqslant i_{0}, \\
u_{i+r} & \text { for } i_{0}<i \leqslant r+t .\end{cases}
\end{aligned}
$$

Our assumptions are made in such a way that $T^{\prime}$ is column-increasing and that $s \circ T^{-1}=s \circ\left(T^{\prime}\right)^{-1}$. It remains to find $i_{0}$. If $s=0$, let $i_{0}=0$. So assume now that $s \geqslant 1$. If $u_{r}<v_{1}$, let $i_{0}=0$. If $v_{s}<u_{r+s+1}$, let $i_{0}=s$. Thus, we can assume that 
$v_{1}<u_{r}$ and $u_{r+s+1}<v_{s}$. The latter inequality shows that $u_{r+s-1}<u_{r+s+1}<v_{s}$, taking into account the fact that $T$ is column-increasing. In particular, we must have $s \geqslant 2$, since for $s=1$, we get a contradiction. Consider the pairs $\left(u_{r+i-1}, v_{i}\right)$. For $i=1$, we have $u_{r}>v_{1}$; for $i=s$, we have $u_{r+s-1}<v_{s}$, and thus there is some $i_{0}$ with $1 \leqslant i_{0}<s$ such that

$$
u_{r+i_{0}-1}>v_{i_{0}} \text { and } u_{r+i_{0}}<v_{i_{0}+1} .
$$

This completes the proof.

Note that this lemma yields the implication (iii) $\Rightarrow$ (i) in Theorem 1. Let $w$ be condensed. According to $\S 5.3$, there exists an $\varepsilon(w)$-tableau $T$ with $\omega(T)=w$. If $\pi$ is arbitrary with $\pi \leqslant \varepsilon(w)$, then the lemma shows the existence of a $\pi$-tableau $T^{\prime}$ with $\omega\left(T^{\prime}\right)=\omega(T)=w$.

5.5. We are going to reverse the considerations of the previous lemma. Let $T$ be a column-increasing $\pi$-tableau. A crossing of $T$ is a pair $(a, i, j),\left(a^{\prime}, i^{\prime}, j^{\prime}\right)$ of squares of $\pi$ belonging to different columns (that is, $\left.(a, j) \neq\left(a^{\prime}, j^{\prime}\right)\right)$ such that the following conditions are satisfied:

(1) the length condition: $i \geqslant i^{\prime}, \tilde{\pi}_{j}^{(a)}-i \leqslant \bar{\pi}_{j^{\prime}}^{\left(a^{\prime}\right)}-i^{\prime}$;

(2) the type condition: $a^{\prime}+i^{\prime} \equiv a+i+1(\bmod n)$;

(3) the value condition: $T(a, i, j)<T\left(a^{\prime}, i^{\prime}, j^{\prime}\right), T\left(a^{\prime}, i^{\prime}-1, j^{\prime}\right)<T(a, i+1, j)$. If we write $u_{t}=T(a, t, j)$ for $1 \leqslant t \leqslant m=\tilde{\pi}_{j}^{(a)}$, and $v_{t}=T\left(a^{\prime}, t, j^{\prime}\right)$ for $1 \leqslant t \leqslant$ $m^{\prime}=\tilde{\pi}_{j^{\prime}}^{\left(a^{\prime}\right)}$, we may visualize a crossing as in Fig. 4 , where again squares on the same horizontal level are supposed to have the same type. Since we allow $i=m$, and $i^{\prime}=1$, there are also the possibilities shown in Figs 5 and 6 . (The name crossing should indicate that we are looking for sequences of squares on which $T$ increases and that going down columns, we may change from one column to the other. This is what will be done in the proof of the next lemma.)

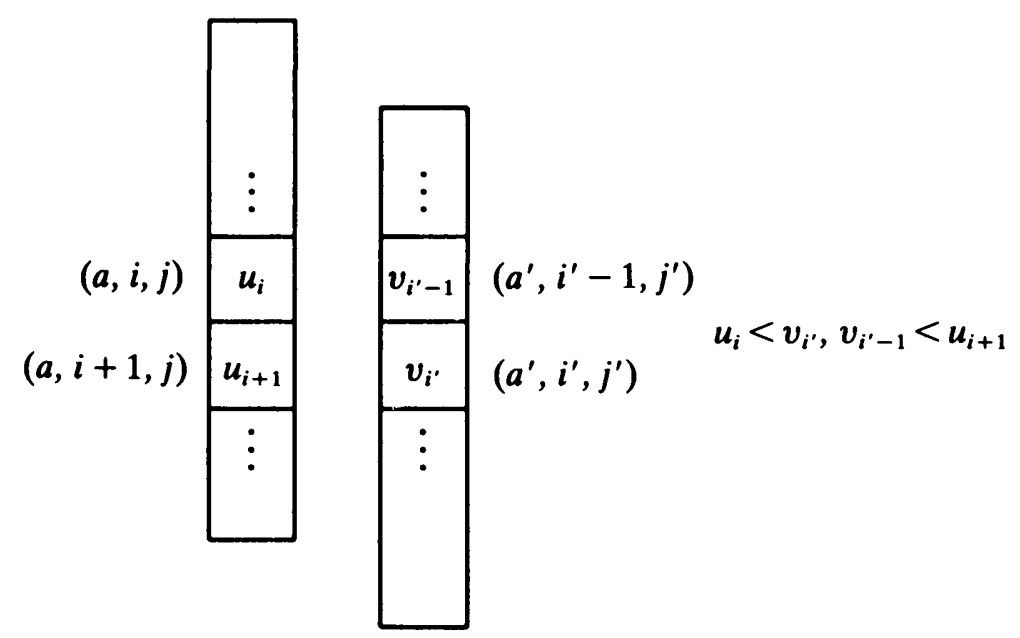

Fig. 4

Lemma. Let $T$ be a column-increasing $\pi$-tableau with a crossing. Then there exists $\lambda$ with $\lambda<\pi$ and a column-increasing $\lambda$-tableau $T^{\prime}$ such that $\omega\left(T^{\prime}\right)=\omega(T)$. 


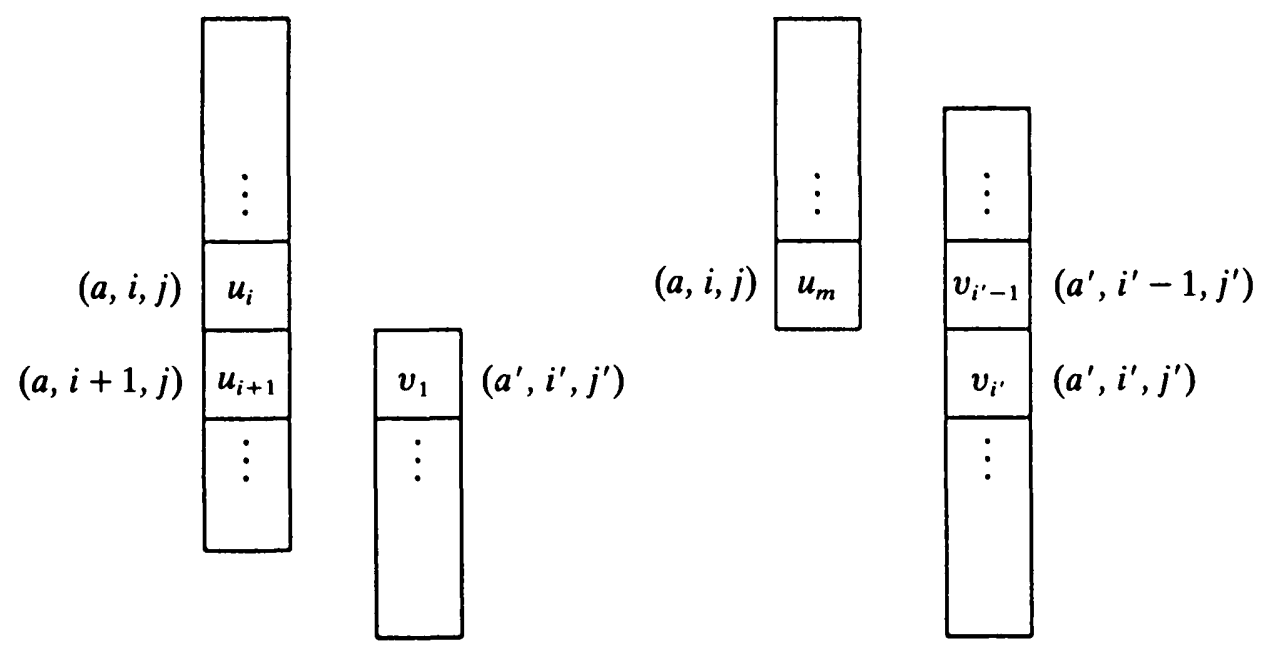

FIG. 5

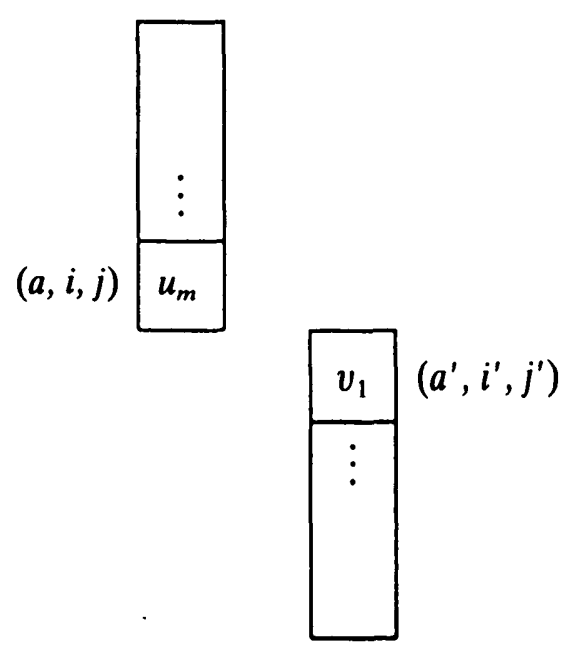

Fig. 6

Proof. Let $(a, i, j),\left(a^{\prime}, i^{\prime}, j^{\prime}\right)$ be squares of $\pi$, where a crossing occurs. Let $m=\tilde{\pi}_{j}^{(a)}, m^{\prime}=\tilde{\pi}_{j^{\prime}}^{\left(a^{\prime}\right)}$. Let $\mu$ be obtained from $\pi$ by deleting the $j$ th column of $\pi^{(a)}$ and the $j^{\prime}$ th column of $\pi^{\left(a^{\prime}\right)}$. Let

$$
r:=i-i^{\prime}+1, \quad s:=m-r, \quad t:=m^{\prime}-s=\left(\tilde{\pi}_{j^{\prime}}^{\left(a^{\prime}\right)}-i^{\prime}\right)-\left(\tilde{\pi}_{j}^{(a)}-i\right)+1 .
$$

Then $r \geqslant 1, s \geqslant 0, t \leqslant 1$, and $m=r+s, m^{\prime}=s+t$. In order to obtain $\lambda$, we add to $\mu^{(a)}$ a column of length $r+s+t$ and to $\mu^{\left(a^{\prime}\right)}$ a column of length $s$. Thus $\lambda<\pi$. The $\lambda$-tableau $T^{\prime}$ is defined as follows: it should coincide with $T$ on those columns which are not changed. Let $u_{t}=T(a, t, j)$, for $1 \leqslant t \leqslant m$, and $v_{t}=T\left(a^{\prime}, t, j^{\prime}\right)$ for $1 \leqslant t \leqslant m^{\prime}$. The values of $T^{\prime}$ going down the new column of $\lambda^{(a)}$ of length $r+s+t$ shall be $u_{1}, u_{2}, \ldots, u_{i}, v_{i^{\prime}}, v_{i^{\prime}+1}, \ldots, v_{m^{\prime}}$, and going down the new column of $\lambda^{\left(a^{\prime}\right)}$ of length $s$, these values shall be $v_{1}, v_{2}, \ldots, v_{i^{\prime}-1}, u_{i+1}, u_{i+2}, \ldots, u_{m}$. The value condition implies that $T^{\prime}$ is column-increasing. Also, $s \circ T^{-1}=s \circ\left(T^{\prime}\right)^{-1}$, and thus $\omega(T)=\omega\left(T^{\prime}\right)$. 
5.6. It remains to study $\pi$-tableaux without crossings.

LeMMA. Let $\pi$ be an $n$-tuple of partitions. If there exists a column-increasing $\pi$-tableau without crossings, then $\pi$ is separated.

Proof. Let $T$ be a column-increasing $\pi$-tableau. Assume that there is some $t$ such that any $\pi^{(a)}$ has a column of length $t$, say let $\tilde{\pi}_{j(a)}^{(a)}=t$ for $1 \leqslant a \leqslant n$. Choose $a$ such that $T(a, 1, j(a))$ is minimal; in particular, we have

$$
T(a, 1, j(a))<T(\overline{a+1}, 1, j(\overline{a+1})) .
$$

But then $(a, 1, j(a)),(\overline{a+1}, 1, j(\overline{a+1}))$ is a crossing.

Proposition. Let $w$ be a condensed word and $\pi$ an $n$-tuple of partitions. Assume that there is a column-increasing $\pi$-tableau $T$ with $\omega(T)=w$ and that no column-increasing $\pi$-tableau $T^{\prime}$ with $\omega\left(T^{\prime}\right)=w$ has a crossing. Then $\pi=\varepsilon(w)$.

Proof. Let $w=s_{1} s_{2} \ldots s_{n}$ with $s_{i} \in \mathbb{Z} / n \mathbb{Z}$. Also let $\varphi=(\varphi(1), \ldots, \varphi(h))$ be a tower of periodic functions with $\varphi(h) \neq 0$ such that $w=\omega(\varphi)$. Let

$$
I_{h}=\{z-1, \ldots, z-h(n-1)\}
$$

and assume $\varphi(h)_{a} \neq 0$, for some $z-1 \leqslant a \leqslant z-n+1$. We want to show that the first column of $\pi^{(\bar{a})}$ has length $h$.

Let $e=e(\varphi)$. For $1 \leqslant t \leqslant h$, let $a_{t}=a+(t-1)(n-1)$, and let $u_{t}=\sum_{i>a_{t}} e_{i}$. The periodicity of $\varphi(h)$ asserts that $\varphi(h)_{a_{t}} \geqslant 1$; thus $e_{a_{t}} \geqslant 1$. It follows that $s_{u_{t}+1}=\bar{a}_{t}$.

Let $h^{\prime}$ be the length of the first column of $\pi^{(a)}$. By induction on $t$, we show that $h^{\prime} \geqslant t$ and we construct a column-increasing $\pi$-tableau $T_{t}$ such that $T_{t}(\bar{a}, i, 1)=$ $u_{i}+1$, for $1 \leqslant i \leqslant t$, and $\omega\left(T_{t}\right)=w$.

First, consider the case $t=1$. Let $T^{-1}\left(u_{1}+1\right)=(b, i, j)$, and assume that $i>1$. Since the square $(b, i, j)$ is of type $\bar{a}$, the square $(b, i-1, j)$ is of type $\overline{a-1}$. Since $T$ is column-increasing, $1 \leqslant T(b, i-1, j) \leqslant u_{t}$. Thus $s_{T(b, i-1, j)}$ is one of the letters $s_{1}, s_{2}, \ldots, s_{u_{t}}$, and all of them belong to $\{\overline{z-1}, \overline{z-2}, \ldots, \overline{a+2}, \overline{a+1}\}$, a contradiction. Therefore, $i=1$ and $b=\bar{a}$. Replace $T$ by a standard $\pi$-tableau $T_{1}=T \circ \sigma$, where $\sigma$ is a permutation of $\|\pi\|$ which fixes the rows. Then $T_{1}(\bar{a}, 1,1)=u_{1}+1$.

Consider now the cases $t \geqslant 2$. Let

$$
\left(a_{1}, i_{1}, j_{1}\right),\left(a_{2}, i_{2}, j_{2}\right), \ldots,\left(a_{s}, i_{s}, j_{s}\right), \ldots
$$

be the squares of $\|\pi\|$ of type $\overline{a+t-1}$ such that $T_{t-1}\left(a_{s}, i_{s}, j_{s}\right)>u_{t}$, and $T_{t-1}\left(a_{s}, i_{s}-1, j_{s}\right) \leqslant u_{t}$. We assume that we have ordered them in such a way that $i_{1} \geqslant i_{2} \geqslant \ldots \geqslant i_{s} \geqslant \ldots$, and such that for $i_{s}=i_{s+1}$, we have

$$
\tilde{\pi}_{j_{s}}^{\left(a_{s}\right)}-i_{s} \geqslant \tilde{\pi}_{j_{s+1}}^{\left(a_{s+1}\right)}-i_{s+1} .
$$

Note that $(*)$ is actually satisfied for all $s \geqslant 1$. Namely, if $i_{s}>i_{s+1}$, and $\tilde{\pi}_{j_{s}}^{\left(a_{s}\right)}<\tilde{\pi}_{j_{s+1}}^{\left(a_{s+1}\right)}$, then the two squares $\left(a_{s}, i_{s}-1, j_{s}\right)$ and $\left(a_{s+1}, i_{s+1}, j_{s+1}\right)$ form a crossing for $T_{t-1}$.

Next, let us show that $i_{1} \leqslant t$. Let $m=i_{1}-1$ and $u_{i}=T_{t-1}\left(a_{1}, i, j_{1}\right)$, for $1 \leqslant i \leqslant m$. Then $v:=s_{u_{1}} s_{u_{2}} \ldots s_{u_{m}}$ is an indecomposable subword of $s_{1} s_{2} \ldots s_{u_{i}}$. The scooping height of $s_{1} s_{2} \ldots s_{u_{t}}$ is at most $t$, and thus $m \leqslant t$. But if we assume that 
$m=t$, then $s_{u_{1}}=\overline{a-1}$, but $\overline{a-1}$ does not occur in $s_{1}, \ldots, s_{u_{1}}$. Thus $v$ is an indecomposable subword of $s_{u_{1}+1} s_{u_{1}+2} \ldots s_{u_{t}}$ and this is of scooping height at most $t-1$, and then $i_{1}-1=m \leqslant t-1$.

Consider now the first column of $\pi^{(\bar{a})}$. By assumption, we know that $T(\bar{a}, i, 1)=u_{i}+1$, for $1 \leqslant i \leqslant t-1$; thus $T(\bar{a}, t-1,1) \leqslant u_{t}$. When the square $(\bar{a}, t, 1)$ exists, it is of type $\overline{a+t-1}$, and also $T_{t-1}(\bar{a}, t, 1)>u_{t}$, since otherwise we get an indecomposable subword of $s_{1} s_{2} \ldots s_{u_{t}}$, which cannot exist.

Assume now that $i_{1}<t$. Then the square $(\bar{a}, t, 1)$ does not exist, since otherwise $(\bar{a}, t, 1)$ would be one of our squares $\left(a_{s}, i_{s}, j_{s}\right)$; thus $i_{s}=t$ for some $s$, and this contradicts our ordering $i_{1} \geqslant i_{2} \geqslant \ldots$. But then $(\bar{a}, t-1,1)$ and $\left(a_{1}, i_{1}, j_{1}\right)$ is a crossing for $T_{t-1}$, again a contradiction. This shows that $i_{1}=t$. Now, $i_{1}=t$ implies that $a_{1}=\bar{a}$; thus the column through $\left(a_{1}, i_{1}, j_{1}\right)$ belongs to $\pi^{(\bar{a})}$, and therefore $h^{\prime} \geqslant \tilde{\pi}_{j_{1}}^{(\bar{a})}$. In particular, the square $(\bar{a}, t, 1)$ exists, and thus it is one of the squares $\left(a_{s}, i_{s}, j_{s}\right)$, and we must have $\tilde{\pi}_{1}^{(\bar{a})} \geqslant h^{\prime}$; thus $\tilde{\pi}_{1}^{(\bar{a})}=h^{\prime}$. Since we have $i_{1}=t$ and $\tilde{\pi}_{1}^{(\bar{a})}-i_{1}=h^{\prime}-t$, we see that we can assume $\left(a_{1}, j_{1}\right)=(\bar{a}, 1)$.

We consider now the squares $\left(a_{s}, i, j_{s}\right)$ with $i \geqslant i_{s}$ and $s \geqslant 1$, as the squares of a partition $l$ with first row

and $k$ th row

$$
\left(a_{1}, i_{1}, j_{1}\right),\left(a_{2}, i_{2}, j_{2}\right), \ldots
$$

$$
\left(a_{1}, i_{1}+k-1, j_{1}\right),\left(a_{2}, i_{2}+k-1, j_{2}\right), \ldots .
$$

The restriction of $T_{t-1}$ to $\|l\|$ is a column-increasing $l$-tableau, and we use the reordering lemma in order to change this $l$-tableau to a standard $l$-tableau. (Note that here the value set is not the canonical one.) Now $T_{t}$ is obtained from $T_{t-1}$ by keeping the values on the squares which do not belong to $\|l\|$ and using the values of the standard $l$-tableau for the squares in $\|l\|$. The square $T_{t-1}^{-1}\left(u_{t}+1\right)$ clearly is of the form $\left(a_{s}, i_{s}, j_{s}\right)$, and thus in the first row of $\|l\|$, and $u_{t}+1$ is the lowest value which occurs under $T_{t-1}$ on $\|l\|$. It follows that $T_{t}\left(a_{1}, i_{1}, j_{1}\right)=u_{t}+1$, but $\left(a_{1}, i_{1}, j_{1}\right)=(\bar{a}, t, 1)$; thus $T_{t}(\bar{a}, t, 1)=u_{t}+1$, as required.

5.7. Let $T$ be a column-increasing $\pi$-tableau. Let $\omega(T)=s_{1} s_{2} \ldots s_{|\pi|}$, with $s_{i} \in \mathbb{Z} / n \mathbb{Z}$. The restriction of $T$ to any column yields an indecomposable subword of $\omega(T)$, more precisely, the restriction to a column of type $a[h]$ yields a subword of the form $a[h]$ : consider the $j$ th column of $\pi^{(a)}$, with vertices $(a, i, j),(a, 2, j), \ldots,(a, h, j)$, where $h=\tilde{\pi}_{j}^{(a)}$. Then

$$
s_{T(a, 1, j)} s_{T(a, 2, j)} \ldots s_{T(a, h, j)}=a[h],
$$

and since $T$ is column-increasing, this is a subword of $\omega(T)$. Since $T$ is bijective, different columns yield disjoint subsets $\left\{T(a, i, j) \mid 1 \leqslant i \leqslant \tilde{\pi}_{j}^{(a)}\right\}$ of $\{1,2, \ldots,|\pi|\}$. Thus we see that $\omega(T)$ is a merging of the various indecomposable words $a\left[\tilde{\pi}_{j}^{(a)}\right]$.

5.8. Theorem 2. Let $w$ be a condensed word, let $\pi=\varepsilon(w)$. Then all columnincreasing $\pi$-tableaux $T$ with $\omega(T)=w$ are equivalent.

Remark. Let $w=\omega(\varphi)$ for some tower of periodic functions $\varphi$, and $\pi=\varepsilon(w)$. We have constructed in $\S 5.3$ a standard $\pi$-tableau $T_{\varphi}$ with $\omega\left(T_{\varphi}\right)=w$. Thus, the theorem asserts that any $\pi$-tableau $T$ with $\omega(T)=w$ is equivalent to $T_{\varphi}$. Note, that in general, there may be other standard $\pi$-tableaux $T$ with $\omega(T)=w$; for 
example, let $w=12^{2}$ and $\pi=((1,1),(1))$. Then both $\pi$-tableaux $T$ with $\omega(T)=$ $w$ are standard:

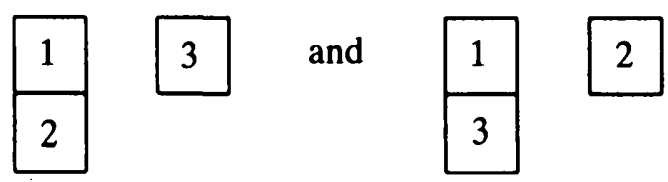

Of course, they are equivalent. On the other hand, for $\pi^{\prime} \neq \varepsilon(w)$, there may exist a unique standard $\pi^{\prime}$-tableau $T$ with $\omega(T)=w$ and a $\pi^{\prime}$-tableau $T^{\prime}$ with $\omega\left(T^{\prime}\right)=w$ which is not equivalent to $T$. For example, let $w=121$ and $\pi=((2),(1))$, and consider the $\pi$-tableaux

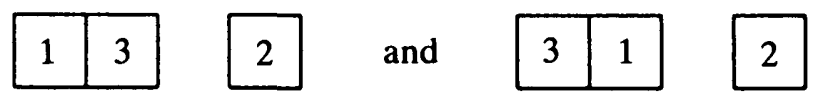

Proof of Theorem 2. Let $\varphi=(\varphi(1), \ldots, \varphi(h))$ be a tower of periodic functions $\varphi(t): I_{t} \rightarrow \mathbb{N}_{0}$, for $1 \leqslant t \leqslant h$, with $\varphi(h) \neq 0$, so that $w=\omega(\varphi)$. Let $e=e(\varphi)$. Let $\pi=\varepsilon(\varphi)=\varepsilon(w)$. Let $T$ be a $\pi$-tableau with $\omega(T)=w$. We want to show that $T$ is equivalent to $T_{\varphi}$. Let $I_{h}=\{a-1, \ldots, a-h(n-1)\}$. Let $i$ be minimal with $\varphi(h)_{a-i} \neq 0$.

First, consider the case $a-i \notin I_{h-1}$. We may assume $i=1$, otherwise we shift $I_{h}$ accordingly. Since $a-1 \in I_{h} \backslash I_{h-1}$, we have $e_{a-1}=\varphi(h)_{a-1}$. Let $m=\varphi(h)_{a-1}$. By construction of $\pi=\varepsilon(\varphi)$, there are $m$ columns of length $h$ in $\pi^{(\overline{a-1})}$, and these are the only columns of $\pi^{(a-1)}$ of length greater than or equal to $h$; thus they have column-index $1,2, \ldots, m$. We claim that $T(\overline{a-1}, 1, j) \leqslant m$ for $1 \leqslant j \leqslant m$. Assume on the contrary, that $T(\overline{a-1}, 1, j)>m$ for some $j$, with $1 \leqslant j \leqslant m$. Since $s(\overline{a-1}, 1, j) \equiv a-1$, and

$$
w=(\overline{a-1})^{e_{a-1}}(\overline{a-2})^{e_{a-2}} \ldots(\overline{a-h(n-1)})^{e_{a-h(n-1)}},
$$

it follows that $T(\overline{a-1}, 1, j)>\sum_{i=1}^{n} e_{a-i}$. If we write $w=s_{1} s_{2} \ldots s_{|\pi|}$, with $s_{i} \in$ $\mathbb{Z} / n \mathbb{Z}$, the subword

$$
S_{T(\overline{a-1}, 1, j)} S_{T(\overline{a-1}, 2, j)} \ldots S_{T(\overline{a-1}, h, j)}
$$

is an indecomposable subword of $v=(\overline{a-n-1})^{e_{a-n-1}} \ldots(\overline{a-h(n-1)})^{e_{a-h(n-1)}}$. However, $v$ is of scooping height at most $h-1$, so it cannot have an indecomposable subword of length $h$. This contradiction shows that $T(\overline{a-1}, 1, j) \leqslant m$ for $1 \leqslant j \leqslant m$, and therefore $T(\overline{a-1}, 1,-)$ is a permutation of $\{1,2, \ldots, m\}$. It follows that $T$ is equivalent to a $\pi$-tableau $\bar{T}$ with $\bar{T}(\overline{a-1}, 1, j)=j$ for all $1 \leqslant j \leqslant v$, and such that $T$ and $\bar{T}$ coincide on the remaining squares. Let $\varphi^{\prime}=\left(\varphi^{\prime}(1), \ldots, \varphi^{\prime}(h)\right)$ be the tower of periodic functions with $\varphi^{\prime}(t): I_{t} \rightarrow \mathbb{N}_{0}$ and $e\left(\varphi^{\prime}\right)_{1}=0, e\left(\varphi^{\prime}\right)_{i}=e_{i}$, for $i \neq 1$. Clearly, $\varphi^{\prime}$ exists and is uniquely determined, and we have $\omega\left(\varphi^{\prime}\right)=s_{2}^{e_{2}} \ldots s_{m}^{e_{m}}$. Let $\pi^{\prime}=\varepsilon\left(\varphi^{\prime}\right)$ and $w^{\prime}=\omega\left(\varphi^{\prime}\right)$. Note that $\pi^{\prime}$ is obtained from $\pi$ by deleting the $v$ columns of type $\overline{a-1}[h]$ and adding $v$ columns of type $\bar{a}[h-1]$. Since $\pi$ has no column of type $\bar{a}[h]$, the same is true for $\pi^{\prime}$; thus we may consider the new columns of $\pi^{\prime}$ of type $\bar{a}[h-1]$ as the first $v$ columns of $\pi^{\prime(\bar{a})}$. We may embed $\left[\pi^{\prime}\right]$ into $[\pi]$ by sending a square of the form $(\bar{a}, i, j)$ with $1 \leqslant j \leqslant v$ to $(\overline{a-1}, i+1, j)$, and the remaining ones to their counterparts in $[\pi]$. In this way, $\bar{T}$ (or $T$ ) gives rise to a column-increasing $\pi^{\prime}$-tableau denoted by $T^{\prime}$, 
with value set $\{v+1, v+2, \ldots,|\pi|\}$, and $\omega\left(T^{\prime}\right)=w^{\prime}$. By induction, $T^{\prime}$ is equivalent to $T_{\varphi^{\prime}}$, and this obviously implies that $T$ is equivalent to $T_{\varphi}$.

Second, consider the case $a-i \in I_{h-1}$. Then $a-i-(h-1)(n-1)$ cannot belong to $I_{h-1}$, and $\varphi(h)_{a-i-(h-1)(n-1)} \neq 0$. Let $i^{\prime}$ be maximal with $\varphi(h)_{a-i^{\prime}} \neq 0$. Since $a-i^{\prime} \notin I_{h-1}$, we have $e_{a-i^{\prime}}=\varphi(h)_{a-i^{\prime}}$, and we set $v=\varphi(h)_{a-i^{\prime}}$. There are $v$ columns of length $h$ with socle of type $a-i^{\prime}$, and $T$ takes on these socle squares the values $|\pi|-v+1, \ldots,|\pi|$. An argument similar to the one used in the first case shows that, up to equivalence, we can assume that the value on the $j$ th column of length $h$ with socle of type $\overline{a-i^{\prime}}$ is precisely $|\pi|-v+j$. And again we use induction in order to show that $T$ is equivalent to $T_{\varphi}$. Here, $\pi^{\prime}$ is obtained from $\pi$ by deleting the squares $T^{-1}(u)$ with $|\pi|-v+1 \leqslant u \leqslant|\pi|$. This completes the proof.

\section{The cardinality of $\Pi_{\mathbf{d}}^{s}$}

Recall that we have denoted by $\Pi_{\mathbf{d}}^{s}$ the set of separated $n$-tuples $\pi$ such that $\operatorname{dim} \pi=\mathbf{d}$. Similarly, let $\Pi_{\mathrm{d}}^{n}$ be the set of $n$-tuples $\pi$ of partitions with $\operatorname{dim} \pi=\mathbf{d}$ such that no part of $\tilde{\pi}^{(n)}$ has length divisible by $n$.

\subsection{Proposition. The sets $\Pi_{\mathbf{d}}^{s}, \Pi_{\mathbf{d}}^{n}$ have the same cardinality, for every $\mathbf{d}$.}

Proof. Recall that for any partition $p$, the number of columns of length $h$ is $p_{h}-p_{h+1}$. Given $\pi \in \Pi$, let $m_{h}(\pi)$ be the minimum of the numbers $\pi_{h}^{(a)}-\pi_{h+1}^{(a)}$, for $1 \leqslant a \leqslant n$. Thus $\pi \in \Pi^{s}$ if and only if $m_{h}(\pi)=0$ for all $h$. Also, $\pi \in \Pi^{n}$ if and only if $\pi_{i n}^{(n)}=\pi_{i n+1}^{(n)}$, for all $i \geqslant 1$.

For $t \in \mathbb{N}_{0}$, we define $\Pi(t)$ as the set of all $\pi \in \Pi$ such that $m_{h}(\pi)=0$ for all $h \leqslant t$, and $\pi_{i n}^{(n)}=\pi_{i n+1}^{(n)}$, for all $i>t$. Of course, we have $\Pi(0)=\Pi^{n}$. Also, for $\pi$ of height at most $h$, we see that $\pi \in \Pi(h)$ if and only if $\pi \in \Pi^{s}$. Let $\Pi(t)_{\mathrm{d}}=$ $\Pi(t) \cap \Pi_{d}$; since $\Pi_{d}$ is a finite set, there is some bound $h$ on the height of the elements of $\Pi_{\mathrm{d}}$, and therefore $\Pi(h)_{\mathrm{d}}=\Pi_{\mathrm{d}}^{s}$.

It is sufficient to show that the sets $\Pi(t-1)_{\mathbf{d}}$ and $\Pi(t)_{\mathbf{d}}$ have the same cardinality, for all $t \in \mathbb{N}_{1}$ and all $\mathbf{d} \in \mathbb{N}_{0}^{n}$. We define a bijection $\zeta_{t}: \Pi(t-1) \rightarrow \Pi(t)$ as follows. Given $\pi \in \Pi$, let $m=m_{t}(\pi)$; let $\zeta_{t}(\pi)$ be obtained from $\pi$ by deleting $m$ columns of type $a[t]$, for $1 \leqslant a \leqslant n$, and adding $m$ columns of type $n[t n]$. The columns of length at most $t-1$ coincide for $\pi$ and $\zeta_{t}(\pi)$, and by construction $m_{t}\left(\zeta_{t}(\pi)\right)=0$. Also, the columns of length at least $t n+1$ coincide for $\pi$ and $\zeta_{t}(\pi)$. This shows that for $\pi \in \Pi(t-1)$, we obtain $\zeta_{t}(\pi) \in \Pi(t)$. Conversely, given $\lambda \in \Pi(t)$, let $m^{\prime}=\lambda_{t n}^{(n)}-\lambda_{t n+1}^{(n)}$, the number of columns of type $n[t n]$ in $\lambda$. If we delete the columns of type $n[t n]$ from $\lambda$ and add for $1 \leqslant a \leqslant n$ precisely $m^{\prime}$ columns of type $a[t]$, we obtain some $\pi \in \Pi(t-1)$ with $\zeta_{t}(\pi)=\lambda$. This shows that $\zeta_{t}$ yields a bijection $\Pi(t-1) \rightarrow \Pi(t)$. It is easy to see that $\operatorname{dim} \zeta_{t}(\pi)=\operatorname{dim} \pi$; therefore $\zeta_{t}$ yields a bijection $\Pi(t-1)_{\mathbf{d}} \rightarrow \Pi(t)_{\mathbf{d}}$.

\section{The Lie algebra $\mathrm{n}(n)$}

7.1. Definition. The graph $\tilde{A}_{n-1}$ defines a Lie algebra $\mathfrak{n}(n)$ over $\mathbb{Q}$, as follows: its generators $x_{1}, x_{2}, \ldots, x_{n}$ are indexed by the residue classes of integers modulo 
$n$ (or by integers themselves, again with the convention that $x_{i}=x_{j}$ for $i \equiv j(\bmod n))$, and the defining relations are, for $n \geqslant 3$,

$$
\begin{aligned}
\left(\operatorname{ad} x_{i}\right) x_{j}=0 & \text { for } j \neq i \pm 1(\bmod n), \\
\left(\operatorname{ad} x_{i}\right)^{2} x_{j}=0 & \text { for } j \equiv i \pm 1(\bmod n),
\end{aligned}
$$

whereas for $n=2$, they are

$$
\left(\operatorname{ad} x_{i}\right)^{3} x_{j}=0 \quad \text { for } j \not \equiv i(\bmod 2) .
$$

If $\mathrm{g}$ is the (complex) Kac-Moody Lie algebra of type $\tilde{A}_{n-1}$, with the triangular decomposition $\mathfrak{g}=\mathfrak{n}_{-} \oplus \mathfrak{h} \oplus \mathfrak{n}_{+}$, then $\mathfrak{n}_{+}$is the complexification of $\mathfrak{n}(n)$. Note that $\mathfrak{n}(n)$ is a $\mathbb{Z}^{n}$-graded Lie algebra: denote by $s_{1}, \ldots, s_{n}$ the canonical base elements of $\mathbb{Z}^{n}$, and define the degree of $x_{i}$ to be $s_{i}$. Let $U(\mathfrak{n}(n))$ be the universal enveloping algebra of $\mathfrak{n}(n)$. In addition to $\mathfrak{n}(n), U(\mathfrak{n}(n))$ is also $\mathbb{Z}^{n}$-graded.

It is well-known how to construct a $\mathbb{Q}$-basis for $\mathfrak{n}(n)$. As before, let $\Omega=\Omega(\mathbb{Z} / n \mathbb{Z})$. Recall that words of the form $a[l]=a(\overline{a+1}) \ldots(\overline{a+l-1}) \in \Omega$ with $1 \leqslant a \leqslant n, l \in \mathbb{N}_{1}$, are said to be indecomposable. Any indecomposable word $w$ yields an element $x(w) \in \mathfrak{n}(n)$, as follows: let $x(a[1])=x_{a}$, and define inductively

$$
x(a[l+1])=\left[x_{a}, x((\overline{a+1})[l])\right],
$$

the outer square brackets being the Lie multiplication (for example, for $n=3$, we have $\left.x(2312)=\left[x_{2},\left[x_{3},\left[x_{1}, x_{2}\right]\right]\right]\right)$. Clearly, the degree of $x(a[l])$ is just $\operatorname{dim} a[l]$.

There is the following relation:

$$
\sum_{a=1}^{n} x(a[i n])=0
$$

for all $i \in \mathbb{N}_{1}$. Therefore the elements $x(a[l])$, with $1 \leqslant a \leqslant n$, such that either $a \neq n$ or $l$ is not divisible by $n$, form a generating set of $n(n)$. But it is wellknown that this set is also a $\mathbb{Q}$-basis; therefore we have the following result:

\subsection{Proposition. We have $\operatorname{dim}_{\mathbb{Q}} U(\mathfrak{n}(n))_{\mathbf{d}}=\left|\Pi_{\mathbf{d}}^{n}\right|$, for every $\mathbf{d} \in \mathbb{N}_{0}^{\mathrm{n}}$.}

Proof. Let $J$ be the set of all indecomposable words $a[l]$ with $a \neq n$ or $l$ not divisible by $n$. Note that given $\pi \in \Pi$, we have $\pi \in \Pi^{n}$ if and only if the type of any column of $\pi$ belongs to $J$. Consider $J$ as a totally ordered set using the lexicographical ordering with respect to the natural ordering of $1 \leqslant a \leqslant n$, and the opposite of the natural ordering of $\mathbb{N}_{1}$. For $\pi \in \Pi^{n}$, let $w_{1}, \ldots, w_{m}$ be the types of the columns, first those of $\pi^{(1)}$, ordered as they occur, then those of $\pi^{(2)}$, and so on; in particular, $m=\sum_{a=1}^{n} \pi_{1}^{(a)}$. Since $\pi \in \Pi^{n}$, we have $w_{i} \in J$, and thus $x\left(w_{i}\right)$ is defined. Let $x(\pi)=x\left(w_{1}\right) x\left(w_{2}\right) \ldots x\left(w_{m}\right)$ in $U(\mathfrak{n}(n))$.

Clearly, the set $\left\{x(\pi) \mid \pi \in \Pi^{n}\right\}$ is the Poincaré-Birkhoff-Witt basis with respect to the ordered basis $J$ of $\mathfrak{n}(n)$. Of course, the degree of $x(\pi)$ is $\operatorname{dim} \pi$, and therefore the set $\left\{x(\pi) \mid \pi \in \Pi_{\mathbf{d}}^{n}\right\}$ is a $\mathbb{Q}$-basis of $U(\mathfrak{n}(n))_{\mathbf{d}}$.

Remark. Our investigations will only use the inequality

$$
\operatorname{dim}_{\mathbb{Q}} U(\mathfrak{n}(n))_{\mathbf{d}} \leqslant\left|\Pi_{\mathbf{d}}^{n}\right|,
$$

which relies on the fact that the set $\left\{x(\pi) \mid \pi \in \Pi^{n}\right\}$ is a generating set for $U(\mathfrak{n}(n))$, and thus on the fact that we have the relations $(*)$ for all $i \in \mathbb{N}_{1}$. On the 
other hand, our investigations will imply the equality stated in the proposition; thus we present a new proof that the set $\left\{x(\pi) \mid \pi \in \Pi^{n}\right\}$ is linearly independent.

\section{The composition algebra}

8.1. Let $\mathbb{T}$ be a tube of rank $n$, with End $S(i)$ finite, say $\mid$ End $S(i) \mid=q_{\pi}$; note that $\mid$ End $S(i) \mid$ does not depend on $i$. We denote by $\mathbb{Z}[q]$ the polynomial ring in one indeterminate $q$.

Lemma. Given $w \in \Omega, \pi \in \Pi$, there exists a polynomial $\langle w \mid \pi\rangle \in \mathbb{Z}[q]$ such that the number of composition series of $M(\pi)$ of type $w$ is given by $\langle w \mid \pi\rangle\left(q_{\pi}\right)$.

Proof. We follow closely the proof of Theorem 1 of [6]. (A corresponding statement is always true for representation-finite algebras, and we may replace $\mathbb{T}$ by the full subcategory of direct sums of indecomposable objects of length bounded by some fixed number, thus dealing with a category equivalent to the module category for a representation-finite algebra.) First of all, given $\pi, \lambda \in \Pi$, there is $h(\pi, \lambda) \in \mathbb{N}_{0}$ such that $|\operatorname{Hom}(M(\pi), M(\lambda))|=q_{\pi}^{h(\pi, \lambda)}$. Also, it is easy to see that there is a monic polynomial $\alpha_{\pi}$ such that $\mid$ Aut $M(\pi) \mid=\alpha_{\pi}\left(q_{\pi}\right)$. Next, for $\pi, \lambda \in \mathrm{II}$, there are polynomials $\sigma_{\pi}^{\lambda}, \eta_{\pi}^{\lambda} \in \mathbb{Z}[T]$ such that $\sigma_{\pi}^{\lambda}\left(q_{\pi}\right)$ is the number of submodules of $M(\lambda)$ isomorphic to $M(\pi)$, and $\eta_{\pi}^{\lambda}\left(q_{\pi}\right)$ is the number of submodules $U$ of $M(\lambda)$ with $M(\lambda) / U$ isomorphic to $M(\pi)$. The proof of this assertion is similar to part (4) in the reference mentioned above. Let $w=$ $s_{1} s_{2} \ldots s_{m}$, with $s_{i} \in \mathbb{Z} / n \mathbb{Z}$. Let $w^{\prime}=s_{2} \ldots s_{m}$. Then

$$
\langle w \mid \pi\rangle=\sum_{\pi^{\prime}} \sigma_{\pi^{\prime}}\left\langle w^{\prime} \mid \pi^{\prime}\right\rangle
$$

(we count the number of submodules of $M(\pi)$ isomorphic to $M\left(\pi^{\prime}\right)$, and the number of composition series of $M\left(\pi^{\prime}\right)$ of type $\left.w^{\prime}\right)$. By induction, $\langle w \mid \pi\rangle$ is a polynomial, and it has the desired properties.

EXAMPLE. Let $w=s^{e}$ for some $1 \leqslant s \leqslant n$ and some $e \geqslant 1$. Clearly, $\langle w \mid \pi\rangle=0$ except in the case where $\pi^{(i)}=0$ for $i \neq s$, and $\pi^{(s)}=(1, \ldots, 1)$ with $s$ entries of 1 ; thus $\langle w \mid \pi\rangle=0$ except for $\pi=\varepsilon(w)$. In the exceptional case, $\langle w, \varepsilon(w)\rangle(p)$ is equal to the number of complete flags in an $e$-dimensional vector space over the field with $p$ elements; thus

$$
\langle w, \varepsilon(w)\rangle(p)=\psi_{e}(p)
$$

(for the definition of the polynomial $\psi_{e}(q)$, see the introduction).

8.2. Proposition. Let $w$ be condensed, say $w=s_{1}^{e_{1}} s_{2}^{e_{1}} \ldots s_{t}^{e_{t}}$, with $s_{i} \in \Delta_{0}$, $s_{i} \neq s_{i+1}$, and $e_{i} \geqslant 1$ for all $i$. Then

$$
\langle w, \varepsilon(w)\rangle=\prod_{i=1}^{t} \psi_{e_{i}}\left(q_{\pi}\right) .
$$

Proof. By Theorem 2, there is precisely one equivalence class of columnincreasing $\varepsilon(w)$-tableaux $T$ with $\omega(T)=w$. Therefore, by Proposition 2.3, the 
module $M(\varepsilon(w))$ has a unique reduced filtration of type $w$, and therefore

$$
\langle w, \varepsilon(w)\rangle=\prod_{i=1}^{t}\left\langle s_{i}^{e_{i}}, \varepsilon\left(s_{i}^{e_{i}}\right)\right\rangle=\prod_{i=1}^{t} \psi_{e_{i}}\left(q_{\pi}\right),
$$

by the example above.

8.3. Proposition. If $w$ is a condensed word, and $\pi$ an $n$-tuple of partitions with $\langle w, \pi\rangle \neq 0$, then $\pi \leqslant \varepsilon(w)$.

This is the consequence of Theorem 1 and Proposition 2.2 which we will need in our further considerations.

Proof. If $\langle w, \pi\rangle \neq 0$, then there is some prime $p$ such that $\langle w, \pi\rangle(p) \neq 0$. Let us consider representations of the cyclic quiver with $n$ vertices over the field with $p$ elements. Since $\langle w, \pi\rangle(p) \neq 0$, the module $M(\pi)$ has a composition series of type $w$; thus, by Proposition 2.2, there is a $\pi$-tableau $T$ with $\omega(T)=w$. Theorem 1 implies that $\pi \leqslant \varepsilon(w)$.

8.4. Let $\mathscr{A}$ be the localization of the polynomial ring $\mathbb{Q}[q]$ at the maximal ideal generated by $q-1$. Thus, $\mathscr{A}$ is a local ring, its maximal ideal is generated by $q-1$, and the residue ring modulo the maximal ideal is isomorphic to $\mathbb{Q}$. Note that all the polynomials $\psi_{e}(q)$ with $e \geqslant 1$ are invertible elements of $\mathscr{A}$.

Recall that $\mathscr{A} \Omega$ is the semigroup algebra of $\Omega=\Omega(\mathbb{Z} / n \mathbb{Z})$; it is the free $\mathscr{A}$-algebra generated by the set $\mathbb{Z} / n \mathbb{Z}$. Also, $\mathscr{A} \Pi$ is the free $\mathscr{A}$-module with basis $\Pi$, and there is the bilinear form

$$
\langle-\mid-\rangle: \mathscr{A} \Omega \times \mathscr{A} \Pi \rightarrow \mathscr{A} .
$$

We have denoted by $\mathscr{R}$ the set of $r \in \mathscr{A} \Omega$ such that $\langle r,-\rangle=0$. It is easy to see that $\mathscr{R}$ is an ideal of $\mathscr{A} \Omega$ (see [7]), and $\mathscr{C}(n)=\mathscr{C}:=\mathscr{A} \Omega / \mathscr{R}$ is called the composition algebra for tubes of rank $n$. Note that $\mathscr{C}$ encodes the knowledge concerning the interrelation between the possible composition series of modules in tubes of rank $n$.

8.5. Lemma. For every $\pi \in \Pi^{s}$, choose some condensed word $w_{\pi}$ such that $\varepsilon\left(w_{\pi}\right)=\pi$. The set $\left\{w_{\pi} \mid \pi \in \Pi^{s}\right\}$ is the basis of a free $\mathscr{A}$-submodule of $\mathscr{A} \Omega$ which intersects $\mathscr{R}$ in zero.

Proof. Consider a finite sum $x=\sum_{\lambda \in \Pi^{s}} a_{\lambda} w_{\lambda}$ in $\mathscr{A} \Omega$, with $a_{\pi} \in \mathscr{A}$, and assume $x \in \mathscr{R}$. We claim that all $a_{\pi}=0$. Suppose this is not the case, choose $\pi$ maximal with respect to the ordering $\leqslant$ such that $a_{\pi} \neq 0$, and consider

$$
\langle x, \pi\rangle=\sum_{\lambda} a_{\lambda}\left\langle w_{\lambda}, \pi\right\rangle .
$$

On the one hand, $\langle x, \pi\rangle=0$, since $x \in \mathscr{R}$. On the right-hand side, for $\lambda \neq \pi$, we cannot have $\pi \leqslant \varepsilon\left(w_{\pi}\right)=\lambda$, by the maximality of $\pi$. Therefore $\left\langle w_{\lambda}, \pi\right\rangle=0$ by Proposition 8.3. But $\left\langle w_{\pi}, \pi\right\rangle \neq 0$, according to Proposition 8.2, and therefore the right-hand side is non-zero, a contradiction.

8.6. Our aim is a description of $\mathscr{C}$ by generators and relations. We again denote the residue class of $x_{i} \in \Delta_{0} \subseteq \Omega$ modulo $\mathscr{R}$ by $x_{i}$. 
Proposition. For $n \geqslant 3$, the elements $x_{i}$ in $\mathscr{C}$ satisfy the relations

$$
{ }^{+} \rho_{2}\left(x_{i}, x_{i+1}\right)=0, \quad \rho_{2}^{+}\left(x_{i+1}, x_{i}\right)=0,
$$

for all $1 \leqslant i \leqslant n$, and $\rho_{1}\left(x_{i}, x_{j}\right)=0$ for all $j \neq i \pm 1(\bmod n)$.

For $n=2$, the elements $x_{1}, x_{2}$ in $\mathscr{C}$ satisfy the relations

$$
\rho_{3}\left(x_{1}, x_{2}\right)=0, \quad \rho_{3}\left(x_{2}, x_{1}\right)=0 .
$$

Proof. Since $n \geqslant 2$, we have $\operatorname{Ext}^{1}(S(i), S(i))=0$ for all $i$. First, consider $n \geqslant 3$; thus $\operatorname{Ext}^{1}(S(i+1), S(i))=0$ for all $i$, and therefore the fundamental relations as established in [7] are valid.

Let $n=2$. The following table exhibits the polynomial $\langle w \mid \pi\rangle$ for all $w, \pi$ with $\operatorname{dim} w=(1,3)=\operatorname{dim} \pi$ :

\begin{tabular}{l|cccc} 
& $((0),(2,1,1))$ & $((0),(3,1))$ & $((1,1),(2))$ & $((1),(3))$ \\
\hline$x_{1} x_{2}^{3}$ & 0 & 0 & $(q+1)\left(q^{2}+q+1\right)$ & $(q+1)\left(q^{2}+q+1\right)$ \\
$x_{2} x_{1} x_{2}^{2}$ & $q+1$ & $q+1$ & $(q+1)^{2}$ & $(q+1)\left(q^{2}+q+1\right)$ \\
$x_{2}^{2} x_{1} x_{2}$ & $q+1$ & $(q+1)^{2}$ & $q+1$ & $(q+1)\left(q^{2}+q+1\right)$ \\
$x_{2}^{3} x_{1}$ & 0 & $(q+1)\left(q^{2}+q+1\right)$ & 0 & $(q+1)\left(q^{2}+q+1\right)$
\end{tabular}

and it follows that $q \rho_{3}\left(x_{2}, x_{1}\right)=0$. Of course, $q$ is invertible in $\mathscr{A}$, and thus $\rho_{2}\left(x_{2}, x_{1}\right)=0$. By symmetry, we also have the other equality.

8.7. TheOREM 3. For $n \geqslant 3$, the composition algebra $\mathscr{C}(n)$ is generated over $\mathscr{A}$ by $x_{1}, \ldots, x_{n}$ with relations

$$
{ }^{+} \rho_{2}\left(x_{i}, x_{i+1}\right)=0=\rho_{2}^{+}\left(x_{i+1}, x_{i}\right)
$$

for $1 \leqslant i \leqslant n$, and $\rho_{1}\left(x_{i}, x_{j}\right)=0$ for $j \not i \pm 1(\bmod n)$.

The composition algebra $\mathscr{C}(2)$ is generated over $\mathscr{A}$ by $x_{1}, x_{2}$, with relations

$$
\rho_{3}\left(x_{1}, x_{2}\right)=0=\rho_{3}\left(x_{2}, x_{1}\right) .
$$

Proof. Let $\mathscr{C}^{\prime}(n)=\mathscr{A} \Omega / \mathscr{R}^{\prime}$; for the definition of $\mathscr{R}^{\prime}$ see the introduction. As we have seen, $\mathscr{R}^{\prime} \subseteq \mathscr{R}$; thus there is a canonical surjective algebra homomorphism $\eta: \mathscr{C}^{\prime}(n) \rightarrow \mathscr{C}(n)$. We consider $\mathscr{A} \Omega$ as a $\mathbb{Z}^{n}$-graded algebra, the degree of $x_{i}$ being $s_{i}$, where $s_{1}, \ldots, s_{n}$ is the canonical basis of $\mathbb{Z}^{n}$. Note that for $w \in \Omega$, the degree of $w$ is just $\operatorname{dim} w$. It is easy to see that $\mathscr{R}$ is a homogeneous ideal, since for $w \in \Omega$ and $\pi \in \Pi$, we have $\langle w \mid \pi\rangle \neq 0$ only in the case where $\operatorname{dim} w=\operatorname{dim} \pi$. Also, the defining relations for $\mathscr{C}^{\prime}(n)$ are homogeneous; thus both $\mathscr{C}^{\prime}(n)$ and $\mathscr{C}(n)$ are $\mathbb{Z}^{n}$-graded, and $\eta$ preserves the grading. We may identify $\mathscr{C}^{\prime}(n) /\langle q-1\rangle$ with $U(\mathrm{n}(n))$, since $\mathscr{C}^{\prime}(n) /\langle q-1\rangle$ is the free $\mathbb{Q}$-algebra with generators $x_{1}, \ldots, x_{n}$ and the corresponding relations (obtained from $\rho_{1},{ }^{+} \rho_{2}, \rho_{2}^{+}$and $\rho_{3}$ by replacing $q$ by 1 ).

For $\mathbf{d} \in \mathbb{N}_{0}^{n}$, we want to see that the canonical map $\eta_{\mathrm{d}}: \mathscr{C}^{\prime}(n)_{\mathrm{d}} \rightarrow \mathscr{C}(n)_{\mathrm{d}}$ is bijective. Now, $\mathscr{C}^{\prime}(n)_{\mathrm{d}}$ is a finitely generated $\mathscr{A}$-module, its radical is

$$
(q-1)^{\mathscr{C}^{\prime}}(n)_{\mathrm{d}}
$$

and

$$
\mathscr{C}^{\prime}(n)_{\mathrm{d}} /(q-1) \mathscr{C}^{\prime}(n)_{\mathrm{d}} \cong U(\mathfrak{n}(n))_{\mathrm{d}}
$$


has $\mathbb{Q}$-dimension $\left|\Pi_{\mathrm{d}}^{n}\right|$, according to $\S 7$. By Lemma $8.5, \mathscr{C}(n)_{\mathrm{d}}$ contains a free $\mathscr{A}$-submodule of rank $\left|\Pi_{\mathrm{d}}^{s}\right|$. Since $\left|\Pi_{\mathrm{d}}^{s}\right|=\left|\Pi_{\mathrm{d}}^{n}\right|$ by $\S 5$, it follows that $\eta_{\mathrm{d}}$ is bijective.

8.8. Recall that $\mathscr{S}$ is the set of $y \in \mathscr{A} \Pi$ such that $\langle-, y\rangle=0$. Two elements $y, y^{\prime}$ of $\mathscr{A} \Pi$ will be called composition equivalent provided $\langle-, y\rangle=\left\langle-, y^{\prime}\right\rangle$, thus, if and only if $y-y^{\prime} \in \mathscr{S}$.

THEOREM 4. For every $\pi \in \Pi^{s}$, choose some condensed word $w_{\pi}$ such that $\varepsilon\left(w_{\pi}\right)=\pi$. The set $\left\{w_{\pi} \mid \pi \in \Pi^{s}\right\}$ is the basis of a free $\mathscr{A}$-submodule of $\mathscr{A} \Omega$ and this submodule is a direct complement for $\mathscr{R}$. Also, $\mathscr{A} \Pi=\mathscr{A} \Pi^{s} \oplus \mathscr{S}$.

The last assertion implies that every $\pi \in \Pi$ is composition equivalent to an $\mathscr{A}$-linear combination of separated $n$-tuples of partitions.

Proof. First, we show that $\mathscr{A} \Pi^{s} \cap \mathscr{S}=0$. Consider a finite sum $y=\sum_{\lambda \in \Pi^{s}} a_{\lambda} \lambda$ in $\mathscr{A} \Pi^{s}$ with $a_{\lambda} \in \mathscr{A}$, and assume $y \in \mathscr{S}$. We claim that all $a_{\lambda}=0$. Suppose this is not so, choose $\pi$ minimal with respect to the ordering $\leqslant$ such that $a_{\pi} \neq 0$, and consider

$$
\left\langle w_{\pi}, y\right\rangle=\sum_{\lambda \in \Pi^{s}} a_{\lambda}\left\langle w_{\pi}, \lambda\right\rangle .
$$

On the one hand, $\left\langle w_{\pi}, y\right\rangle=0$, since $y \in \mathscr{S}$. On the right-hand side, for $\lambda \neq \pi$, we cannot have $\lambda \leqslant \varepsilon\left(w_{\pi}\right)=\pi$, by the minimality of $\pi$. Therefore $\left\langle w_{\pi}, \lambda\right\rangle=0$ by Proposition 8.3. But $\left\langle w_{\pi}, \pi\right\rangle \neq 0$ according to Proposition 8.2, and therefore the right-hand side is non-zero, a contradiction.

The proof of Theorem 3 has shown that $\mathscr{C}(n)_{\mathrm{d}}$ is a free $\mathscr{A}$-module of rank $\left|\Pi_{\mathrm{d}}^{s}\right|$. Assume $y \in \mathscr{A} \Pi$ and $\operatorname{dim} y=\mathrm{d}$. We claim that $y \in \mathscr{S}$ if and only if $\left\langle w_{\pi}, y\right\rangle=0$ for all $\pi \in \Pi_{\mathbf{d}}^{s}$. For the proof, assume $\left\langle w_{\pi}, \pi\right\rangle=0$ for $\pi \in \Pi_{\mathbf{d}}^{s}$. The set $\left\{w_{\pi} \mid \pi \in \Pi_{\mathbf{d}}^{s}\right\}$ goes under the canonical map $(\mathscr{A} \Omega)_{\mathrm{d}} \rightarrow \mathscr{C}(n)_{\mathrm{d}}$ to the basis of a free $\mathscr{A}$-submodule of rank $\left|\Pi_{\mathrm{d}}^{s}\right|$. Thus, given $x \in(\mathscr{A} \Omega)_{\mathrm{d}}$, there is some $0 \neq a \in \mathscr{A}$ and some $x^{\prime} \in \mathscr{R}$ such that $a x-x^{\prime}$ is an $\mathscr{A}$-linear combination of the elements $w_{\pi}$ with $\pi \in \Pi_{\mathrm{d}}^{s}$. Therefore $a\langle x, y\rangle=\langle a x, y\rangle=\left\langle a x-x^{\prime}+x^{\prime}, y\right\rangle=0$. But $\mathscr{A}$ has no zero divisors, and thus $\langle x, y\rangle=0$. This shows that $y \in \mathscr{S}$.

In order to show that $\mathscr{A} \Pi^{s}+\mathscr{S}=\mathscr{A} \Pi$, let $y \in(\mathscr{A} \Pi)_{\mathrm{d}}$. Let $\Pi^{s}(y)$ be the set of all $\pi \in \Pi_{\mathbf{d}}^{s}$ such that $\left\langle w_{\pi}, y\right\rangle \neq 0$ for some $\pi^{\prime} \leqslant \pi$. By induction on $\left|\Pi^{s}(y)\right|$, we show that $y \in \mathscr{A} \Pi^{s}+\mathscr{S}$. If $\Pi^{s}(y)=\varnothing$, then we have seen above that $y \in \mathscr{S}$. Assume, $\Pi^{s}(y) \neq \varnothing$, and choose $\pi \in \Pi^{s}(y)$ minimal with respect to $\leqslant$. Let

$$
y^{\prime}=y-\frac{\left\langle w_{\pi}, y\right\rangle}{\left\langle w_{\pi}, \pi\right\rangle} \pi \text {. }
$$

Note that $\left\langle w_{\pi}, \pi\right\rangle$ is invertible in $\mathscr{A}$. Then $\Pi^{s}\left(y^{\prime}\right) \subseteq \Pi^{s}(y)$, and $\pi \notin \Pi^{s}\left(y^{\prime}\right)$. By induction, $y^{\prime} \in \mathscr{A} \Pi^{s}+\mathscr{S}$, and thus also $y \in \mathscr{A} \Pi^{s}+\mathscr{S}$.

Altogether, we see that $\mathscr{A} \Pi=\mathscr{A} \Pi^{s} \oplus \mathscr{S}$. In particular, given $x \in \mathscr{A} \Omega$, we see that $x \in \mathscr{R}$ if and only if $\langle x, \pi\rangle=0$ for all $\pi \in \Pi^{s}$. Consider an element $x \in(\mathscr{A} \Omega)_{\mathrm{d}}$. We want to show that $x$ is the sum of an element from $\mathscr{R}$ and an $\mathscr{A}$-linear combination of elements $w_{\pi}$ with $\pi \in \Pi^{s}$. Let $\Pi^{s}(x)$ be the set of $\pi \in \Pi_{d}^{s}$ such that $\left\langle x \mid \pi^{\prime}\right\rangle \neq 0$, for some $\pi^{\prime}$ with $\pi \leqslant \pi^{\prime}$. By induction on $\left|\Pi^{s}(x)\right|$, we show that $x$ is the sum of an element from $\mathscr{R}$ and an $\mathscr{A}$-linear combination of elements 
$w_{\mu}$ with $\mu \in \Pi^{s}$. If $\Pi^{s}(x)=\varnothing$, then $x \in \mathscr{R}$. Assume $\Pi^{s}(x) \neq \varnothing$, and choose $\pi \in \Pi^{s}(x)$ maximal with respect to $\leqslant$. Let

$$
x^{\prime}=x-\frac{\langle x, \pi\rangle}{\left\langle w_{\pi}, \pi\right\rangle} w_{\pi} ;
$$

note that $\left\langle w_{\pi}, \pi\right\rangle$ is invertible in $\mathscr{A}$. Then $\Pi^{s}\left(x^{\prime}\right) \subseteq \Pi^{s}(x)$, and $\pi \notin \Pi^{s}\left(x^{\prime}\right)$. By induction, $x^{\prime}$ is the sum of an element from $\mathscr{R}$ and an $\mathscr{A}$-linear combination of elements $w_{\mu}$ with $\mu \in \Pi^{s}$. Therefore the same is true for $x$. This completes the proof.

Remark. For $\pi \in \Pi^{s}$, we have chosen a condensed word $w_{\pi}$ such that $\varepsilon\left(w_{\pi}\right)=\pi$. The $\mathscr{A}$-submodule of $\mathscr{A} \Omega$ generated by $\left\{w_{\pi} \mid \pi \in \Pi^{s}\right\}$, as well as the set of images $\left\{w_{\pi}+\mathscr{R} \mid \pi \in \Pi^{s}\right\}$ in $\mathscr{C}(n)$, will depend on our choice. For example, let $n=2$, and $d=(1,3)$. Then $\Pi_{\mathbf{d}}^{s}=\{((0),(2,1,1)),((0),(3,1)),((1,1), 2)\}$. All four words $x_{1} x_{2}^{3}, x_{2} x_{1} x_{2}^{2}, x_{2}^{2} x_{1} x_{2}$ and $x_{2}^{3} x_{1}$ are condensed, and $\varepsilon\left(x_{1} x_{2}^{3}\right)=((1,1), 2)$, $\varepsilon\left(x_{2} x_{1} x_{2}^{2}\right)=\varepsilon\left(x_{2}^{2} x_{1} x_{2}\right)=((0),(2,1,1)), \varepsilon\left(x_{2}^{3} x_{1}\right)=((0),(3,1))$. For $w_{((0),(2,1,1))}$ we may choose either $x_{2} x_{1} x_{2}^{2}$ or $x_{2}^{2} x_{1} x_{2}$. We see that the corresponding $\mathscr{A}$ submodules of $\mathscr{A} \Omega$ generated by $\left\{w_{\pi} \mid \pi \in \Pi^{s}\right\}$ will be different; also $x_{2} x_{1} x_{2}^{2}-$ $x_{2}^{2} x_{1} x_{2}$ does not belong to $\mathscr{R}$ as the table in $\S 8.6$ shows.

\section{Remarks}

9.1. For $\Delta$ a symmetrizable generalized Cartan matrix of affine type, and $\mathfrak{g}=\mathfrak{n}_{-} \oplus \mathfrak{h} \oplus \mathfrak{n}_{+}$a triangular decomposition of the corresponding Kac-Moody algebra, the quantum group $U\left(\mathfrak{h} \oplus \mathfrak{n}_{+}\right)$may always be constructed in terms of composition algebras, as announced in [8]. Consider the case of a symmetric generalized Cartan matrix $\Delta$. Let $\vec{\Delta}$ be a quiver with underlying graph $\Delta$; thus $\vec{\Delta}$ is obtained from $\Delta$ by choosing some orientation. We have to distinguish two cases. First of all, consider the case when $\vec{\Delta}$ has no sink (and no source); then $\Delta$ is a cycle, and we deal with the cyclic orientation. This is the situation investigated in the present paper. Second, in the remaining cases, the path algebra $k \vec{\Delta}$ is finite-dimensional; thus we deal with a finite-dimensional hereditary algebra of tame type. The main tool for determining the structure of the Hall algebra of $k \vec{\Delta}$ is our knowledge of the Auslander-Reiten quiver of $k \vec{\Delta}$. The modules lying in the preprojective, or the preinjective component of $k \vec{\Delta}$ will be handled in the same way as the modules in the cases where $\Delta$ is Dynkin. The main difficulty rests in the modules which belong to tubes, but we may use the results of this paper in order to overcome these difficulties; this will be shown in a forthcoming paper.

9.2. A general investigation of the Hall algebra of a cyclic quiver will be published by Guo [1].

Added in proof, December 1992. Given any graded ring $R=\bigoplus_{g \in \Gamma} R_{g}$ with grading group $\Gamma$ (written additively), a bilinear form $\beta$ in $\Gamma$ with values in $\mathbb{Z}$, and an invertible central element $v \in R_{0}$, one may introduce a new multiplication on the additive group of $R$ by defining

$$
r \circ r^{\prime}=v^{\beta\left(g, g^{\prime}\right)} r r^{\prime}
$$


for $r \in R_{g}, r^{\prime} \in R_{g^{\prime}}$. The algebra $(R) \circ$ obtained in this way is again associative.

In particular, the composition algebra $\mathscr{C}(n)$ studied above is graded with grading group $\mathbb{Z}_{n}=K_{0}(\mathbb{T})$, the Grothendieck group of $\mathbb{T}$ modulo all exact sequences, and there is the Euler characteristic $\varepsilon$ on $K_{0}(\mathbb{T})$. Let

$$
R=\mathscr{C}(n) \otimes_{\mathbb{Z}[q]} \mathbb{Z}\left[q^{\frac{1}{2}}, q^{-\frac{1}{2}}\right], \quad \beta=\varepsilon, \quad \text { and } \quad v=q^{\frac{1}{2}} .
$$

It turns out that $\left(\mathscr{C}(n) \otimes_{\mathbb{Z}|q|} \mathbb{Z}\left[q^{\frac{1}{2}}, q^{-\frac{1}{2}}\right]\right)$ 。 satisfies precisely the Jimbo-Drinfeld relations. Note that the twist of the composition algebra multiplication using the Euler characteristic removes the dependence on the orientation. See the author's paper 'Hall algebras revisited' which will appear in the proceedings of the Israel Conference on Quantum Deformations of Algebras and their Representations, 1991/92.

\section{References}

1. J. Y. Guo, 'The Hall algebra of a cyclic serial algebra', to appear.

2. G. JAMES and A. KERBER, The representation theory of the symmetric group (Addison-Wesley, Reading, Mass., 1981).

3. V. G. KAC, Infinite dimensional Lie algebras, 2nd edn (Cambridge University Press, 1985).

4. G. LuSzTIG, 'Canonical bases arising from quantized enveloping algebras', J. Amer. Math. Soc. 3 (1990) 447-498.

5. I. G. MACDONALD, Symmetric functions and Hall polynomials (Clarendon Press, Oxford, 1979).

6. C. M. Ringel, 'Hall algebras', Topics in algebra, Banach Center Publications 26 (PWN, Warsaw, 1990), pp. 433-447.

7. C. M. RiNGEL, 'Hall algebras and quantum groups', Invent. Math. 101 (1990) 583-592.

8. C. M. RiNGEL, 'From representations of quivers via Hall and Loewy algebras to quantum groups', Proceedings of the Novosibirsk Conference on Algebra 1989, Contemporary Mathematics 131.2 (American Mathematical Society, Providence, R.I., 1992), pp. 381-402.

\section{Fakultät für Mathematik \\ Universität Bielefeld \\ $W-4800$ Bielefeld 1 \\ Germany}

